- STATISTIK

Der Handel zwischen Deutschland und Polen

- LESEHINWEIS

Jahrbuch Polen 2020 Polnische Wirtschaft

STATISTIK

Covid-19 in Polen (Stand: 12. April 2021)

- CHRONIK

$$
\begin{aligned}
& \text { Deutsches } \\
& \text { Polen-Institut }
\end{aligned}
$$




\title{
Robustheit trotz Corona. Die deutsch-polnischen Handelsbeziehungen
}

\author{
Adrian Stadnicki, Ost-Ausschuss der Deutschen Wirtschaft, Berlin
}

DOI: $10.31205 /$ PA.272.01

\section{Zusammenfassung}

Weitgehend unbemerkt von der Öffentlichkeit hat sich Polen 2020 in die Top 5 der deutschen Handelspartner vorgearbeitet. Dass dieser Erfolg in das Krisenjahr der Corona-Pandemie fällt, ist kein Zufall. Er hat vielmehr mit der Stärke Polens als crisis performer zu tun. Die polnische Wirtschaft erwies sich im ersten Jahr der Pandemie im internationalen Vergleich als besonders widerstandsfähig - ähnlich wie schon während der globalen Finanzkrise 2008/2009. Damals war Polen sogar das einzige Land in der EU, das noch ein positives Wirtschaftswachstum verzeichnen konnte. Dies gelang im Corona-Jahr 2020 zwar nicht mehr, aber mit einem Rückgang des Bruttoinlandsprodukts von rund -2,8 Prozent schnitt Polen deutlich besser als der EU-Durchschnitt von -6,3 Prozent ab. Für Deutschland bezifferte sich der Rückgang auf fünf Prozent.

A us Sicht der Handelsbeziehungen gilt: Deutschland ist für Polen am wichtigsten, Polen wird für Deutschland immer wichtiger. Das deutsch-polnische Wirtschaftstandem war selbst im Krisenjahr der CoronaPandemie 2020 ein Erfolgsmodell für Europa und stabilisiert die bilateralen Beziehungen.

\section{Polen überholt Italien}

Im Jahr des 30-jährigen Jubiläums des Deutsch-Polnischen Nachbarschaftsvertrages zählt Polen nunmehr zusammen mit China, den Niederlanden, den USA und Frankreich zu den fünf wichtigsten Handelspartnern der Bundesrepublik weltweit. Mit diesem Sprung nach vorn ereignete sich gleichzeitig im deutschen Außenhandel ein echter Wachwechsel: Polen zog damit an Italien vorbei. Das ist vor dem Hintergrund des Größenunterschiedes zwischen beiden Volkswirtschaften bemerkenswert. Italiens Bruttoinlandsprodukt entspricht fast vier Mal demjenigen Polens, auch die Einwohnerzahl ist mit $60 \mathrm{Mil}-$ lionen deutlich größer als die polnische mit 38 Millionen.

Eine Auswertung der aktuellen Daten des Statistischen Bundesamtes zeigt, dass sich der deutsch-polnische Handel nach einem kurzen, aber heftigen Einbruch im zweiten Quartal 2020 überraschend schnell wieder erholen konnte. Die Lockdowns, Grenzschließungen und Lieferkettenunterbrechungen infolge der Corona-Pandemie wirkten sich damals zunächst massiv auf den bilateralen Warenaustausch aus. Allein im April belief sich der Rückgang gegenüber dem Vorjahr auf 25 Prozent. Doch bis zum Jahresende konnte der deutsch-polnische Handel die zwischenzeitlich dramatischen Verluste nahezu komplett aufholen und erreichte fast noch das Vorjahresniveau von 123 Milliarden Euro. Der Rückgang betrug am Ende nur noch -0,4 Prozent gegenüber 2019.

Im globalen Vergleich zählt Polen damit zu den absoluten Ausnahmen. Der gesamte Warenaustausch der Handelsnation Deutschland erlitt im Jahr 2020 einen Ein- bruch in Höhe von -8,3 Prozent. China war das einzige Land unter den Top 10 der Handelspartner Deutschlands, das ein Plus im Warenaustausch von drei Prozent erreicht hat. Der Handel Deutschlands mit den USA (-9,7 Prozent), Frankreich (-14,5 Prozent) und Italien (-8,4 Prozent) verzeichnete hingegen deutliche Einschnitte. Vor diesem Hintergrund zählt Polen damit zu den wenigen Konjunkturstabilisatoren im deutschen Außenhandel.

Diese enorm wichtige Rolle Polens für die deutsche Wirtschaft sollte auch in der Öffentlichkeit viel stärker gewürdigt werden. Denn Polen erzählt nicht mehr und nicht weniger als eine der großen Erfolgsgeschichten der EU-Osterweiterung von 2004.

\section{$1991-2004-2020$}

1991, im Jahr der Unterzeichnung des deutsch-polnischen Nachbarschaftsvertrages, umfasste der deutschpolnische Handel umgerechnet gerade einmal acht Milliarden Euro. Die Handelsbeziehungen waren bis dahin kaum ausgeprägt, trotz der geographischen Nähe. Zwar bemühte sich der Ost-Ausschuss der Deutschen Wirtschaft seit seiner Gründung 1952 um den Aufbau von Geschäftskontakten, der langjährige Vorsitzende Otto Wolff von Amerongen und der Krupp-Manager Berthold Beitz waren in Warschau gern gesehene Gesprächspartner und gehörten auch beim legendären Besuch von Bundeskanzler Willy Brandt 1970 in Warschau zur deutschen Delegation. Aber der Eiserne Vorhang und die Zugehörigkeit Polens zum Warschauer Pakt erschwerten jahrzehntelang die Geschäftskontakte ebenso wie die nicht kompatiblen Wirtschaftssysteme in West und Ost.

Dies änderte sich mit dem Wendejahr 1989. Nach der Öffnung der polnischen Volkswirtschaft nahm der bilaterale Warenaustausch in den 1990er Jahren Fahrt auf. Bis zum EU-Beitritt Polens 2004 konnte sich so der Warenaustausch über die Oder und Neiße vervierfachen: Im Jahr 2004 belief er sich auf 35 Milliarden Euro. 1991 von Platz 
15 aus gestartet, stand das Land im Jahr 2004 an 11. Stelle in der deutschen Außenhandelsstatistik. Die Aufnahme Polens in den europäischen Binnenmarkt führte dann zu einer rasanten Beschleunigung. Infolge der sich vertiefenden Wirtschaftsbeziehungen und immer engmaschigerer, gemeinsamer Lieferketten kletterte Polen weiter im Ranking der deutschen Handelspartner nach oben.

Im Jahr 2006 wurde im bilateralen Handel die Marke von 50 Milliarden Euro geknackt. Und kurz vor dem Ausbruch der Weltfinanzkrise hatte sich der Warenaustausch im fünften Jahr nach dem EU-Betritt bereits auf 67 Milliarden Euro gegenüber 2004 nochmals verdoppelt.

Die Weltfinanzkrise 2009 ging nicht spurlos am Handel zwischen den beiden Ländern vorbei. Der Rückgang fiel mit -20 Prozent gewaltig aus, das Vorkrisenniveau konnte aber dennoch schnell wieder erreicht werden. Gleichzeitig zog Polen im Jahr 2010 erstmals in die Top 10 der deutschen Handelspartner weltweit ein.

Im deutschen »Osthandel ", also unter den 29 Partnerländern des Ost-Ausschusses der Deutschen Wirtschaft, ist Polen bereits seit dem Jahr 2013 die unangefochtene Nummer eins. Damals konnte das Land erstmals Russland als wichtigsten deutschen Handelspartner in der Region überholen. Parallel mit dem Aufstieg Polens fiel Russland seit dieser Zeit kontinuierlich unter den größten Handelspartnern zurück, sechs Jahre nach der Annexion der Krim und dem Beginn von Wirtschaftssanktionen ist Russland als Handelspartner hinter Tschechien und Ungarn abgerutscht.

2016 kam es dann zu einer historischen Zäsur: Polen übersprang als erstes Land in Mittel- und Osteuropa die magische Marke von 100 Milliarden Euro im Handel mit Deutschland. Und der positive Trend ging weiter: 2019 zog Polen an Großbritannien vorbei auf Platz 6 im Handelsranking. Dieses Überholmanöver kam überraschend früh und hatte zwei Gründe: Die gewohnt hohen Wachstumsraten der polnischen Wirtschaft und das Schwächeln Großbritanniens vor dem Hintergrund des Brexit. Großbritannien ist inzwischen noch weiter zurückgefallen. Das aus der EU ausgetretene Land belegte 2020 nur noch Platz 8 im Ranking der deutschen Außenhandelspartner.

\section{Champion im Handel mit Mittel- und Osteuropa}

Der Warenaustausch mit allen 29 Ländern östlich der Oder belief sich 2020 auf insgesamt 422 Milliarden Euro, was einem Anteil von rund 19 Prozent am deutschen Außenhandel entspricht. Sogar die Weltmächte China und USA kommen zusammen nicht auf diese enorme Summe. Das Volumen im Handel mit diesen beiden Wirtschaftsgiganten beläuft sich "nur" auf 384 Milliarden Euro.

Die Tragweite und Bedeutung des deutschen Osthandels wird inzwischen stark von Polen geprägt, dessen Anteil allein bei rund 30 Prozent liegt. Auf die größte Volkswirtschaft in Osteuropa, die Russische Föderation, entfallen hingegen nur noch elf Prozent. Im vergangenen Jahr ist der deutsch-russische Handel um -22,2 Prozent eingebrochen.

$\mathrm{Zu}$ den wichtigen Playern im deutschen Osthandel zählen neben Polen insbesondere die anderen Mitglieder der Viségrad-Gruppe: die Slowakei, Tschechien und Ungarn. Als Einheit betrachtet, beläuft sich der Warenaustausch der Bundesrepublik mit der V4-Gruppe inzwischen auf 286 Milliarden Euro. Zusammengerechnet ist die Ländergruppe mit ihren 65 Millionen Einwohnern im Außenhandel damit sogar weit wichtiger für die deutsche Wirtschaft als das 1,4 Milliarden Einwohner zählende China (212 Milliarden Euro).

Für alle V4-Länder hat sich der EU-Beitritt $2004 \mathrm{zu}$ einer großen wirtschaftlichen Erfolgsgeschichte entwickelt. Die Länder sind innerhalb kürzester Zeit zu TopPartnern der deutschen Wirtschaft avanciert. Sie stehen inzwischen für 12,8 Prozent des weltweiten Handels der Bundesrepublik, Tendenz steigend. Während Deutschland mit der Slowakei, Tschechien und Ungarn sogar ein Handelsdefizit aufweist, werden nach Polen weiterhin mehr deutsche Güter exportiert, als von dort importiert.

Für alle V4-Länder ist Deutschland der mit Abstand wichtigste Handelspartner. Damit wird auch umgekehrt deutlich, wie stark diese Länder von der deutschen Wirtschaft und der Verlängerung deutscher Wertschöpfungsketten im Zuge der EU-Osterweiterung profitiert haben. Das Beispiel Polens zeigt die enorme Bedeutung der Bundesrepublik aus Sicht der V4 auf: Mit einem Volumen von 123 Milliarden Euro steht Deutschland im Ranking der polnischen Handelspartner mit großem Abstand an erster Stelle. Mit 36 Milliarden Euro folgt die Volksrepublik China auf dem zweiten Platz. Zwischen dem wichtigsten und dem zweitwichtigsten Handelspartner Polens klafft also eine Lücke von bemerkenswerten 87 Milliarden Euro.

Die herausragende Bedeutung Deutschlands lässt sich auch am Anteil an den Gesamtexporten bzw. -importen veranschaulichen: Schätzungen des polnischen Statistischen Hauptamtes (Główny Urząd Statystyczny - GUS) zufolge beläuft sich der Anteil Deutschlands an allen polnischen Exporten auf 29 Prozent und der Anteil an allen Importen auf 22 Prozent.

Aus polnischer Sicht ist zudem das Handelsungleichgewicht mit China interessant: Im Gegensatz zu Deutschland exportiert Polen kaum Güter nach China. Die Ausfuhren in die Volksrepublik fallen mit knapp drei Milliarden Euro gering aus. Im Vergleich dazu werden fast zehn Mal so viele Waren aus China nach Polen importiert. Das Handelsdefizit Polens mit dem Reich der Mitte beläuft sich auf rund 29 Milliarden Euro. Als Absatzmarkt ist China für Polen derzeit kaum relevant.

Ein Blick in die Handelsstatistik mit den übrigen Mitgliedern der Viségrad-Gruppe ist ebenfalls lohnend. Eine 
herausgehobene Stellung kommt hier Tschechien zu. Das Land rangiert bei den polnischen Exporten auf Platz zwei. Die Handelsumsätze mit Ungarn und der Slowakei sind für Polen dagegen nicht von außerordentlicher Bedeutung. Das liegt vor allem an der ähnlichen Wirtschaftsstruktur. Die Länder der Viségrad-Gruppe sind wirtschaftlich auf die größeren westlichen Absatzmärkte in Deutschland, Italien, Frankreich und Großbritannien fokussiert.

Rein ökonomisch betrachtet, steht die Gruppe in einem starken internen Wettbewerb. Die Slowakei, Polen, Tschechien und Ungarn sind bemüht, den Sprung zur innovationsbasierten Wirtschaft möglichst schnell zu schaffen und in den internationalen Wertschöpfungsketten aufzusteigen. Die Länder konkurrieren derzeit nicht mehr um reine Industrieinvestitionen, sondern um Investments im Bereich der Forschung und Entwicklung.

Trotz ihrer politischen Bedeutung zählen sowohl die USA als auch Russland nicht zu den Schwergewichten im polnischen Handel. Entgegen der enormen politischen Distanz zu Russland, ist der polnische Warenaustausch mit dem östlichen Nachbarn (17 Milliarden Euro) deutlich größer als der mit dem NATO-Partner USA (13 Milliarden Euro). Infolge der Diversifizierung der polnischen Gasimporte dürfte Russland allerdings weiter an Bedeutung verlieren.

\section{Besonderheiten in den deutsch-polnischen Wirtschaftsbeziehungen}

Die Stabilität des deutsch-polnischen Handels basiert auch auf dessen Diversität. Sehr viele unterschiedliche Warengruppen passieren die Grenzen, der Erfolg ist auf vielen Schultern verteilt. Das senkt die Anfälligkeit in Krisenzeiten. Zudem sticht keine einzelne Warengruppe besonders heraus, wie dies etwa beim deutschen Handel mit Russland der Fall ist, bei dem Erdgas oder Erdöl eine herausgehobene Stellung einnehmen.

Im Jahr 2019 exportierte Deutschland vor allem chemische Erzeugnisse (14,5 Prozent), Maschinen (14,4 Prozent) sowie Kfz und die dazugehörigen Teile (10,9 Prozent) nach Polen. Textilien, Elektrotechnik und Elektronikgüter spielten ebenfalls eine wichtige Rolle. Polen exportierte umgekehrt Kfz und die dazugehörigen Teile (13,6 Prozent), Maschinen (9,9 Prozent) und Nahrungsmittel nach Deutschland (9,4 Prozent).

Eine Besonderheit in der Warenstruktur ergibt sich im Hinblick auf die polnischen Exporte von Möbeln nach Deutschland. Da Polen der größte Möbelexporteur der Welt ist, nimmt diese Kategorie auch mit einem Anteil von 6,4 Prozent einen relativ wichtigen Platz in den polnischen Exporten nach Deutschland ein.

Die Struktur der Importe und Exporte ähnelt sich ansonsten stark. Das gilt als Indikator für eine enge Verzahnung der beiden Volkswirtschaften. Innerhalb einer recht kurzen Zeitspanne von 30 Jahren wurde Polen eng- maschig in die deutschen Wertschöpfungs- und Lieferketten integriert. Qualität (höhere Wertschöpfung) und Quantität (mehr Handel) dürften weiter zunehmen.

Ausgehend von der engen Verzahnung der beiden Volkswirtschaften ist insbesondere die Entwicklung des Außenhandels im vergangenen Jahr von Interesse. Es stellt sich hier die Frage, wie sich die Lockdowns infolge der Corona-Pandemie mit ihren Grenzschließungen und Lieferkettenunterbrechungen auf die bilateralen Wirtschaftsbeziehungen ausgewirkt haben.

Die jüngsten Daten des Statistischen Bundesamtes ermöglichen eine Auswertung der monatlichen Handelsumsätze des gesamten Jahres 2020. Anhand eines Vergleichs mit dem Vorjahreszeitraum (2019) lassen sich tatsächlich einige Auswirkungen der CoronaEindämmungsmaßnahmen auf den Warenaustausch beobachten. Die ersten Lockdowns und die polnischen Grenzschließungen im März 2020 machten sich in der Handelsstatistik sofort bemerkbar: Im Monat April brach der bilaterale Warenaustausch bereits um 2,5 Milliarden Euro ein, was einem Rückgang von einem Viertel entspricht. Damals kam es infolge von Grenzschließungen durch Polen zeitweise zu LKW-Staus von 40 Kilometern Länge an der Oder-Neiße-Grenze. Die stark rückläufige Tendenz war in ähnlicher Ausprägung noch im Mai zu beobachten. Im Juni verringerte sich dagegen der Einbruch bereits auf 0,6 Milliarden Euro, ehe sich der Handel ab Juli wieder auf dem Vorjahresniveau einpendelte.

Dass es sich dabei um keine Selbstverständlichkeit handelt, zeigt der Vergleich mit Tschechien, das 2019 noch Waren im Wert von 93 Milliarden Euro mit Deutschland handelte. Ähnlich wie Polen meldete Tschechien im ersten Halbjahr nur geringe Infektionszahlen. Dennoch waren die Einschnitte größer und hielten länger an.

Der Warenaustausch mit Tschechien verzeichnete im April 2020 dadurch einen Rückgang von 2,9 Milliarden Euro, was einem Minus von 37 Prozent entspricht. Bis zum Juni waren die Einschnitte noch deutlich spürbar und erst gegen Jahresende, im November, wurden die Monatszahlen des Vorjahres wieder erreicht. Der deutsch-tschechische Handel konnte sich nach einem starken und langen Einbruch letztlich im Gesamtjahr nicht mehr vollständig erholen. Dies dürfte vor allem auf die höhere Abhängigkeit von der Automobilindustrie zurückzuführen sein.

Die deutschen Exporte nach Polen gingen dagegen im Gesamtjahr 2020 mit -1,7 Prozent nur sehr leicht zurück. Aus polnischer Sicht besonders erfreulich: Die deutschen Importe aus Polen legten sogar leicht zu (+1,0 Prozent). Paradoxerweise war "Made in Poland» damit im »Corona-Jahr« gefragter denn je in der Bundesrepublik. Polen exportierte insgesamt Güter im Wert von 58 Milliarden Euro nach Deutschland. 
Betrachtet man das Ranking der Außenhandelspartner nur nach Importen, dann steht Polen sogar an vierter Stelle. Mehr Waren importiert Deutschland nur noch aus China (116 Milliarden Euro), den Niederlanden (88 Milliarden Euro) und den USA (67 Milliarden Euro).

\section{"Polnische Wirtschaft« als Qualitätssiegel}

Das Stereotyp der "polnischen Wirtschaft" als ineffizientem und unorganisiertem Wirtschaftsmodell dürfte angesichts der starken Handelszahlen endgültig widerlegt sein. "Made in Poland" ist inzwischen zu einem Qualitätssiegel avanciert. Produkte aus dem Nachbarland erfreuen sich im qualitätsorientierten Deutschland erkennbar einer besonderen Beliebtheit.

Abschließend stellt sich die Frage nach den Gründen für die polnischen Erfolge: Polen begegnet den wirtschaftlichen Folgen der Corona-Krise aus einer Position der Stärke heraus. Das Land ist die einzige Volkswirtschaft in der Europäischen Union, die seit 1990 ein ununterbrochenes Wachstum verzeichnete. Experten führen dies vor allem auf das antizyklische Kaufverhalten der Polinnen und Polen zurück. In Krisenzeiten werden demnach in Polen vermehrt Güter nachgefragt, während in anderen, eher auf Ersparnisse orientierten Ländern der private Konsum stark einbricht. Das führt dazu, dass die Bevölkerung in Polen sozusagen ein eigenes Konjunkturpaket auflegt und die Wirtschaft stabilisiert.

Die polnischen Wachstumsraten waren auch im Zeitraum vor der Krise mit 4,8 Prozent (2017), 5,4 Prozent (2018) und 4,5 Prozent (2019) sehr hoch und lagen deutlich über dem jeweiligen EU-Durchschnitt. Bereits vor der Krise war Polen damit ein Wachstumsmotor der EU.

Das Wachstum der polnischen Wirtschaft fußt insgesamt auf drei Säulen. Dazu zählen die starke Binnennachfrage (auch in normalen Zeiten, denn die Polinnen und Polen sind konsumfreudig), die auf EU-Fördermitteln beruhenden Investitionen und die steigenden Exporte. Alle drei Faktoren kurbeln das polnische Wachstum an, wobei der private Konsum der stärkste Treiber ist. Die Sozialprogramme der nationalkonservativen Regierung, zu denen auch das Kindergeld 500+ gehört, wirken sich ebenfalls auf den privaten Konsum aus. Hinzu kommen die üppigen Konjunkturpakete der Regierung. Während der Corona-Pandemie wurden mehr als 70 Milliarden Euro an Hilfsgeldern mobilisiert, in seiner Gesamtheit das größte Konjunkturpaket in Mittel- und Osteuropa.

Auch in Zukunft wird die polnische Wirtschaft durch EU-Fördermittel angekurbelt. Warschau dürfte weiterhin ein Top-Profiteur von EU-Kohäsionsgeldern sein. Nach dem gerade verabschiedeten Mehrjährigen Finanzrahmen der EU kann Polen für die Jahre 2021 2027 mit 75 Milliarden Euro aus dem Kohäsionsfonds rechnen. Damit geht jeder fünfte Kohäsionseuro nach Polen. Aus dem europäischen Konjunkturpaket »NextGenerationEU« dürften zudem mehr als 20 Milliarden Euro an weiteren Zuschüssen nach Polen fließen.

\section{Never ending success story? Yes, we can}

Polen ist für Deutschland nicht nur der wichtigste Handelspartner in Mittel- und Osteuropa und ein wirtschaftlicher Impulsgeber, sondern auch ein Stabilisator der deutschen Konjunktur in Krisenzeiten. Das Land ist längst auch keine verlängerte Werkbank mehr, sondern wird immer mehr zu einem Innovationspartner für die deutsche Wirtschaft. Viele deutsche Unternehmen entwickeln ihre digitalen Produkte bereits in Polen und nutzen so die IT-Affinität der im EU-Durchschnitt jungen Bevölkerung.

$\mathrm{Zu}$ den vielversprechenden Signalen zählte im vergangenen Jahr auch, dass der größte Börsengang in Europa mit dem Internetauktionshaus Allegro aus Polen kam. Mit positiven wirtschaftlichen Überraschungen aus Warschau ist also weiter und vermehrt zu rechnen. Allerdings: Die Konkurrenz schläft nicht. Dass der USamerikanische Onlineversandhändler Amazon 2021 erstmals auch in den polnischen Markt eintrat, deutet an, dass die polnischen Unternehmen nun verstärkt auch im globalen Wettbewerb bestehen müssen. Hier scheint eine Vertiefung der Zusammenarbeit mit deutschen Unternehmen sinnvoll, wie sie etwa im Automobilbereich bereits gang und gäbe ist.

Der Ausblick bleibt positiv: Die Megatrends der Digitalisierung und auch der europäische Green Deal dürften die wirtschaftlichen Beziehungen zwischen Polen und Deutschland weiter befeuern. Hier gibt es im traditionellen Kohleland Polen infolge des klimagerechten Umbaus der europäischen Wirtschaft bis 2050 große Potenziale, die gemeinsam gehoben werden können.

Zusammenfassend betrachtet stellen die deutschpolnischen Handelsbeziehungen also eine gemeinsame Erfolgsgeschichte dar. Die Chancen für eine Fortsetzung stehen gut. Wer hätte vor 30 Jahren gedacht, dass Polen im Jubiläumsjahr des deutsch-polnischen Nachbarschaftsvertrages eine derart große Bedeutung für Deutschland erreichen würde? Diese Entwicklung gilt es entsprechend zu würdigen, richtet sich der Fokus der Öffentlichkeit doch in der Regel gern auf die Themen, die beide Länder voneinander trennen. 


\section{Der Handel zwischen Deutschland und Polen}

Grafik 1: Deutschland: Der Handel mit Polen (1991 - 2020; Mrd. Euro)

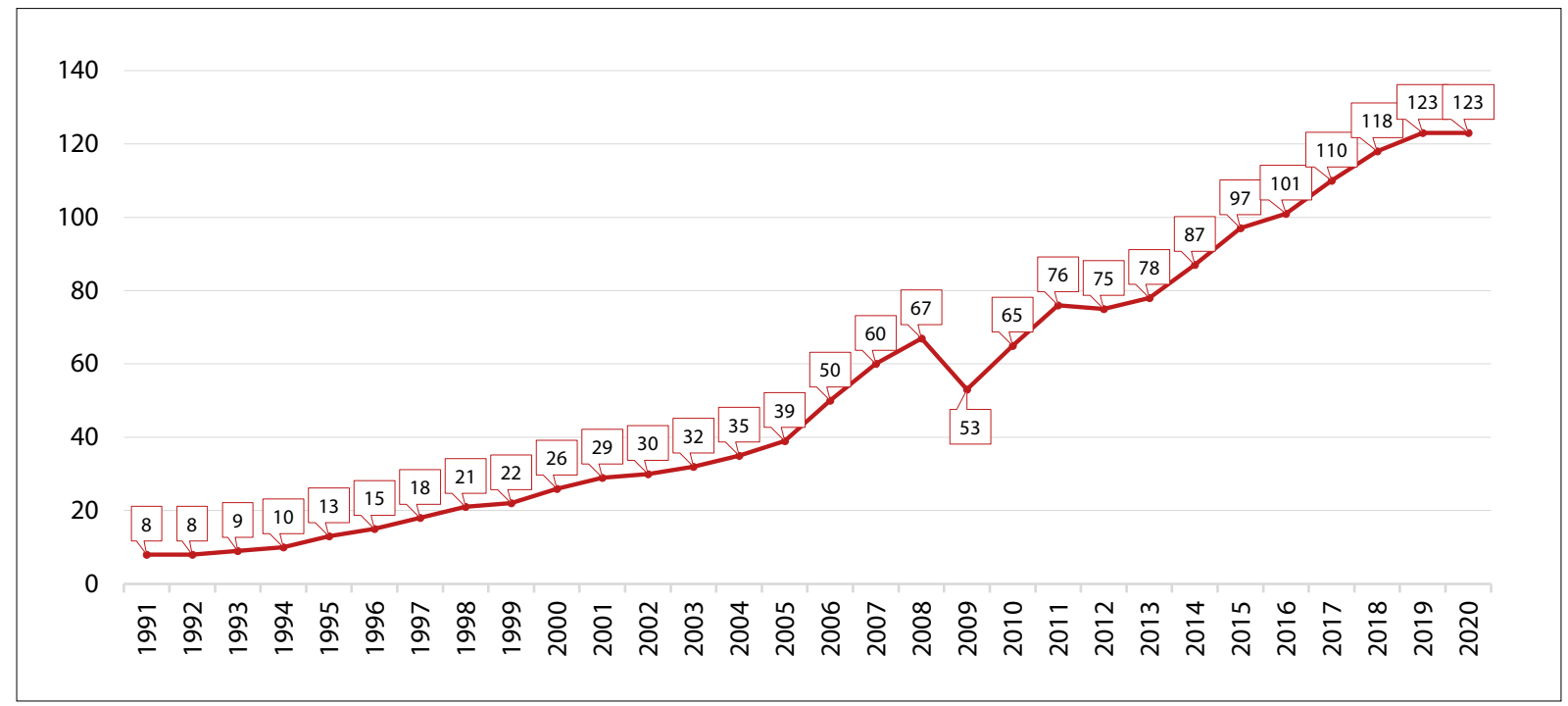

Quelle: Statistisches Bundesamt; Berechnungen Ost-Ausschuss der Deutschen Wirtschaft.

Grafik 2: Deutschland: Der Handel mit Polen und Tschechien (2019, 2020; Mrd. Euro)

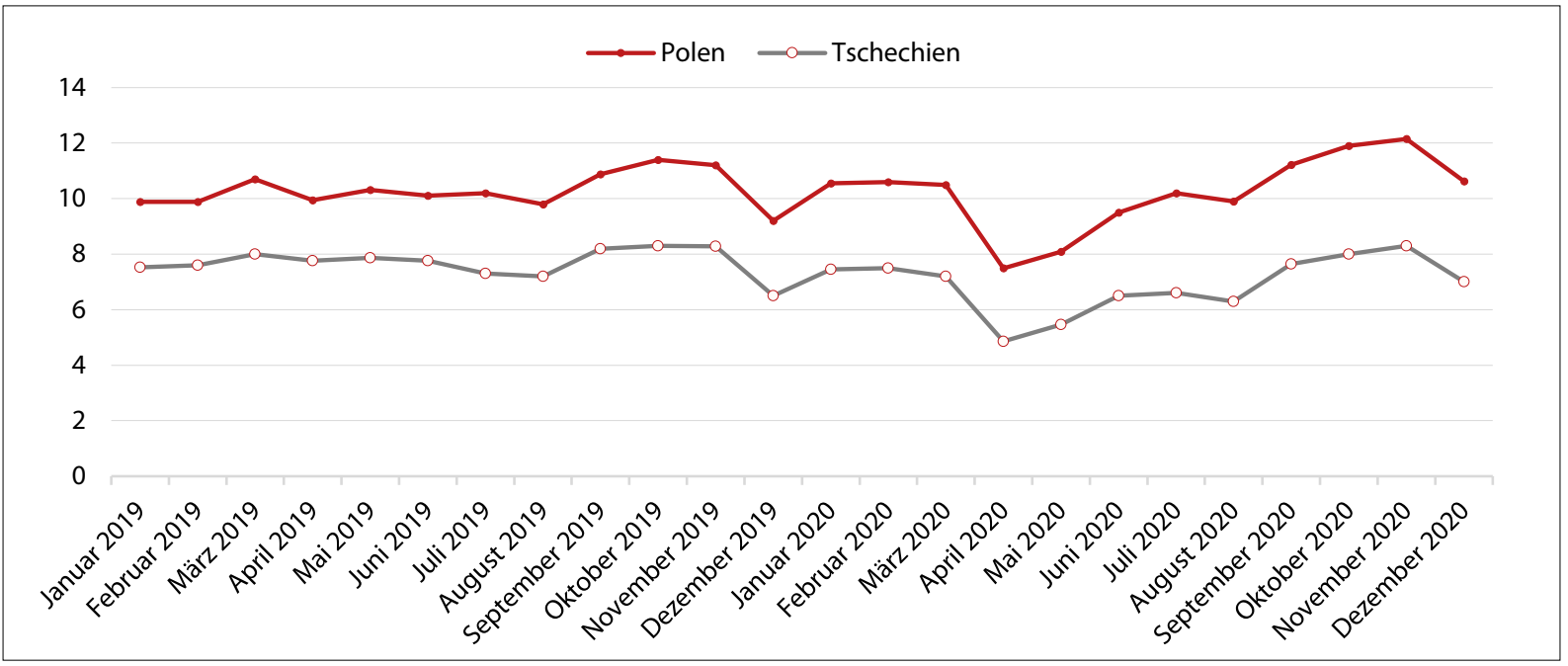

\begin{tabular}{|l|c|c|c|c|c|c|c|c|c|c|c|c|}
\hline & \multicolumn{10}{|c|}{ 2019/Mrd. Euro } \\
\cline { 2 - 14 } & Jan. & Feb. & März & April & Mai & Juni & Juli & Aug. & Sept. & Okt. & Nov. & Dez. \\
\hline Polen & 9,88 & 9,88 & 10,7 & 9,94 & 10,32 & 10,11 & 10,2 & 9,8 & 10,88 & 11,4 & 11,2 & 9,2 \\
\hline Tschechien & 7,52 & 7,6 & 8,0 & 7,77 & 7,87 & 7,76 & 7,3 & 7,2 & 8,2 & 8,3 & 8,29 & 6,5 \\
\hline
\end{tabular}

\begin{tabular}{|c|c|c|c|c|c|c|c|c|c|c|c|c|}
\hline & \multicolumn{12}{|c|}{ 2020/Mrd. Euro } \\
\hline & Jan. & Feb. & März & April & Mai & Juni & Juli & Aug. & Sept. & Okt. & Nov. & Dez. \\
\hline Polen & 10,55 & 10,6 & 10,5 & 7,49 & 8,09 & 9,50 & 10,2 & 9,9 & 11,22 & 11,9 & 12,15 & 10,63 \\
\hline Tschechien & 7,45 & 7,5 & 7,2 & 4,86 & 5,47 & 6,5 & 6,6 & 6,3 & 7,65 & 8,0 & 8,30 & 7,0 \\
\hline
\end{tabular}

Quelle: Statistisches Bundesamt; Berechnungen Ost-Ausschuss der Deutschen Wirtschaft. 


\section{Grafik 3: Deutschland: Ranking der deutschen Handelspartner (2020, in Mrd. Euro und Ranking-Platz)}

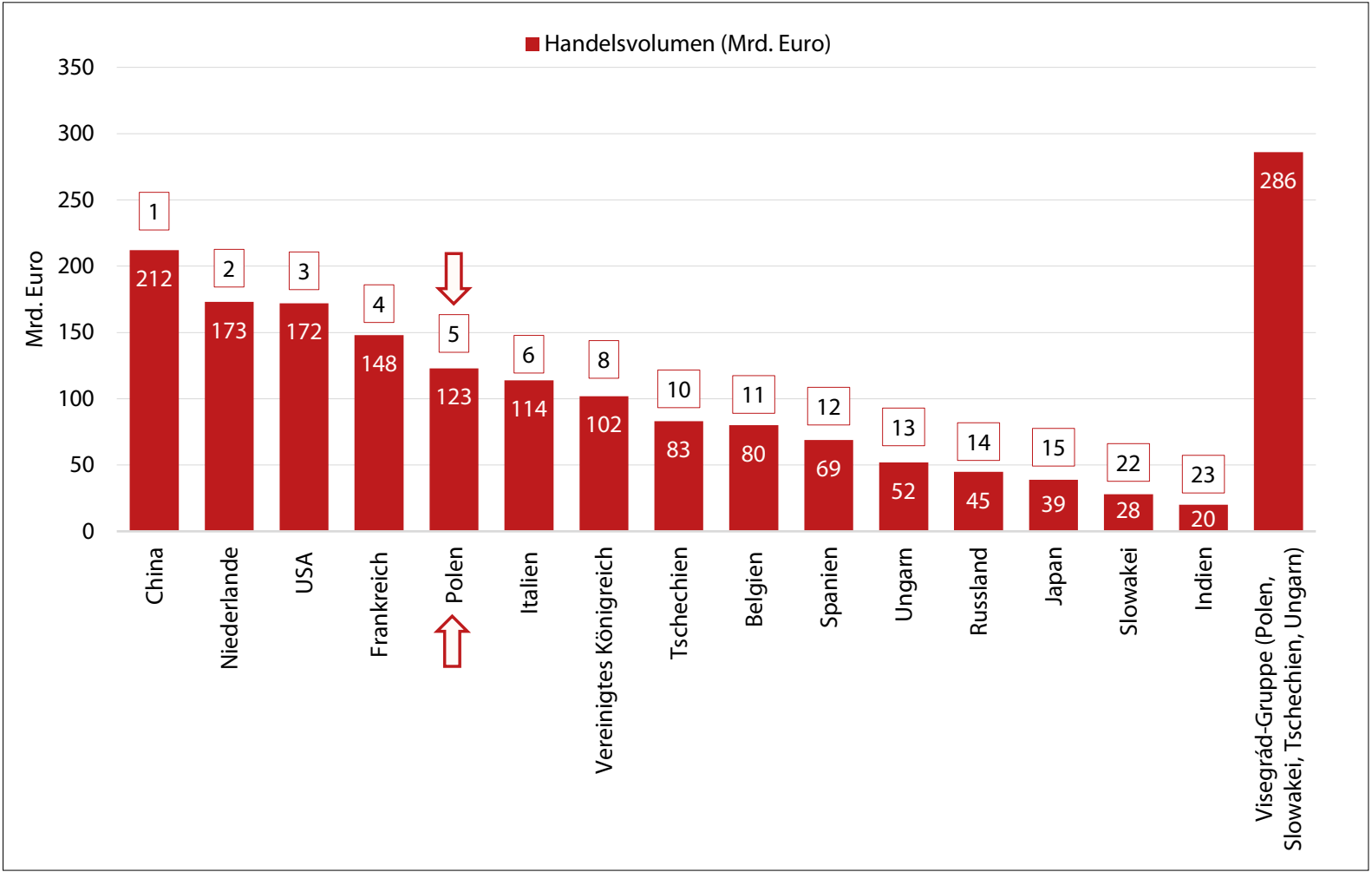

Quelle: Statistisches Bundesamt; Zusammenstellung des Autors Adrian Stadnicki.

Grafik 4: Deutsche Ausfuhrgüter nach Polen 2019*

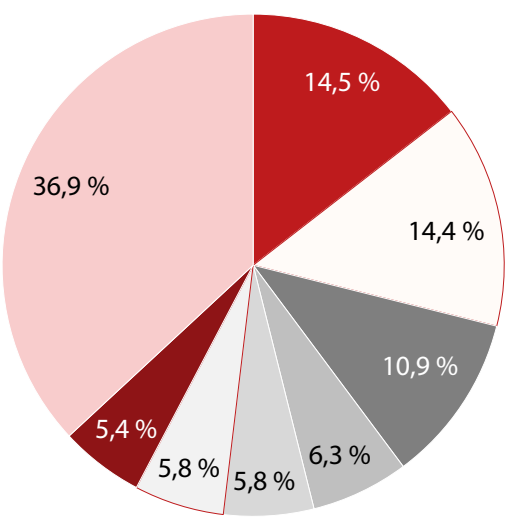

- Chemische Erzeugnisse

$\square$ Maschinen

Kfz und Kfz-Teile

$\square$ Textilien

Elektrotechnik

$\square$ Nahrungsmittel

— Elektronik

Sonstiges

*nach SITC (Standard International Trade Classification)

Quelle: Germany Trade \& Invest; Zusammenstellung des Autors Adrian Stadnicki. 
Grafik 5: Deutsche Einfuhrgüter aus Polen 2019*

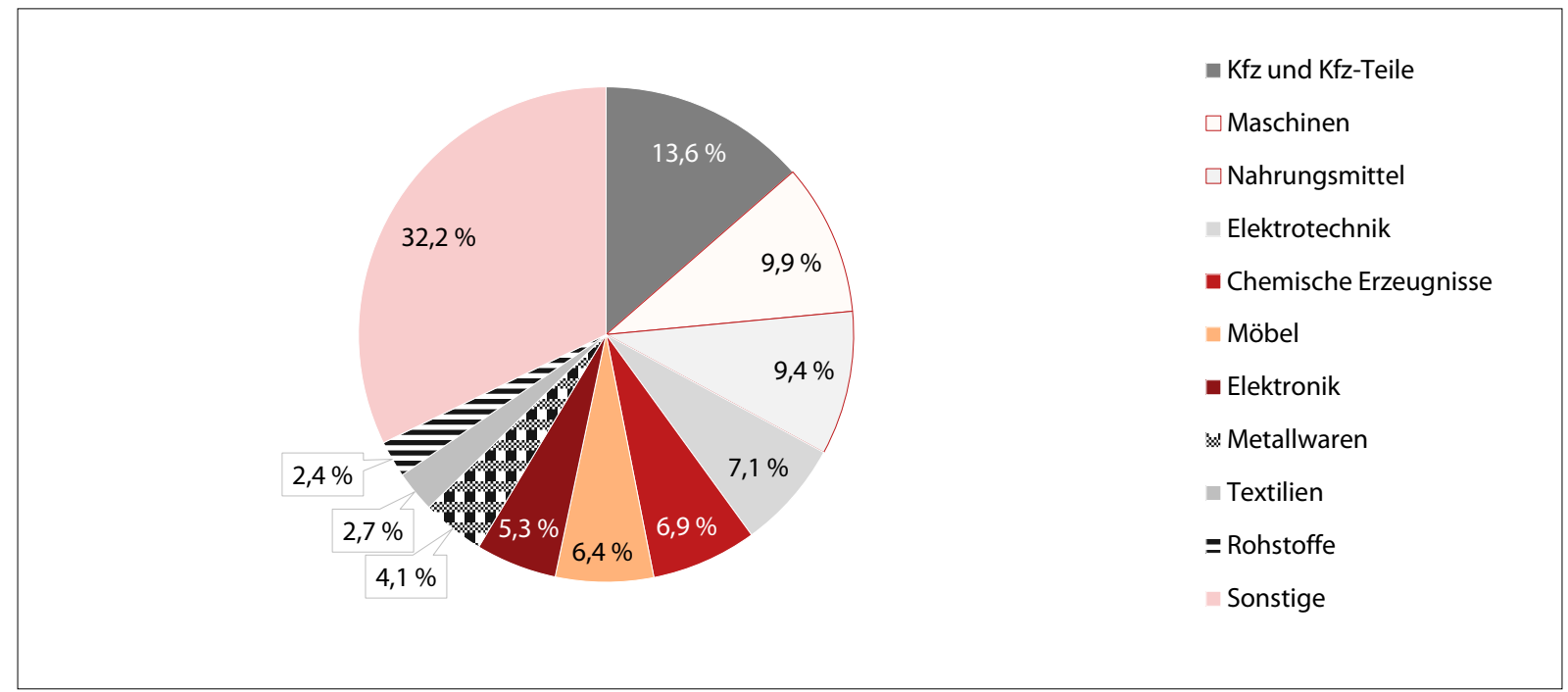

*nach SITC (Standard International Trade Classification)

Quelle: Germany Trade \& Invest; Zusammenstellung des Autors Adrian Stadnicki.

\section{Grafik 6: Polen: Ranking der polnischen Handelspartner (2020, in Mrd. Euro und Ranking-Platz)}

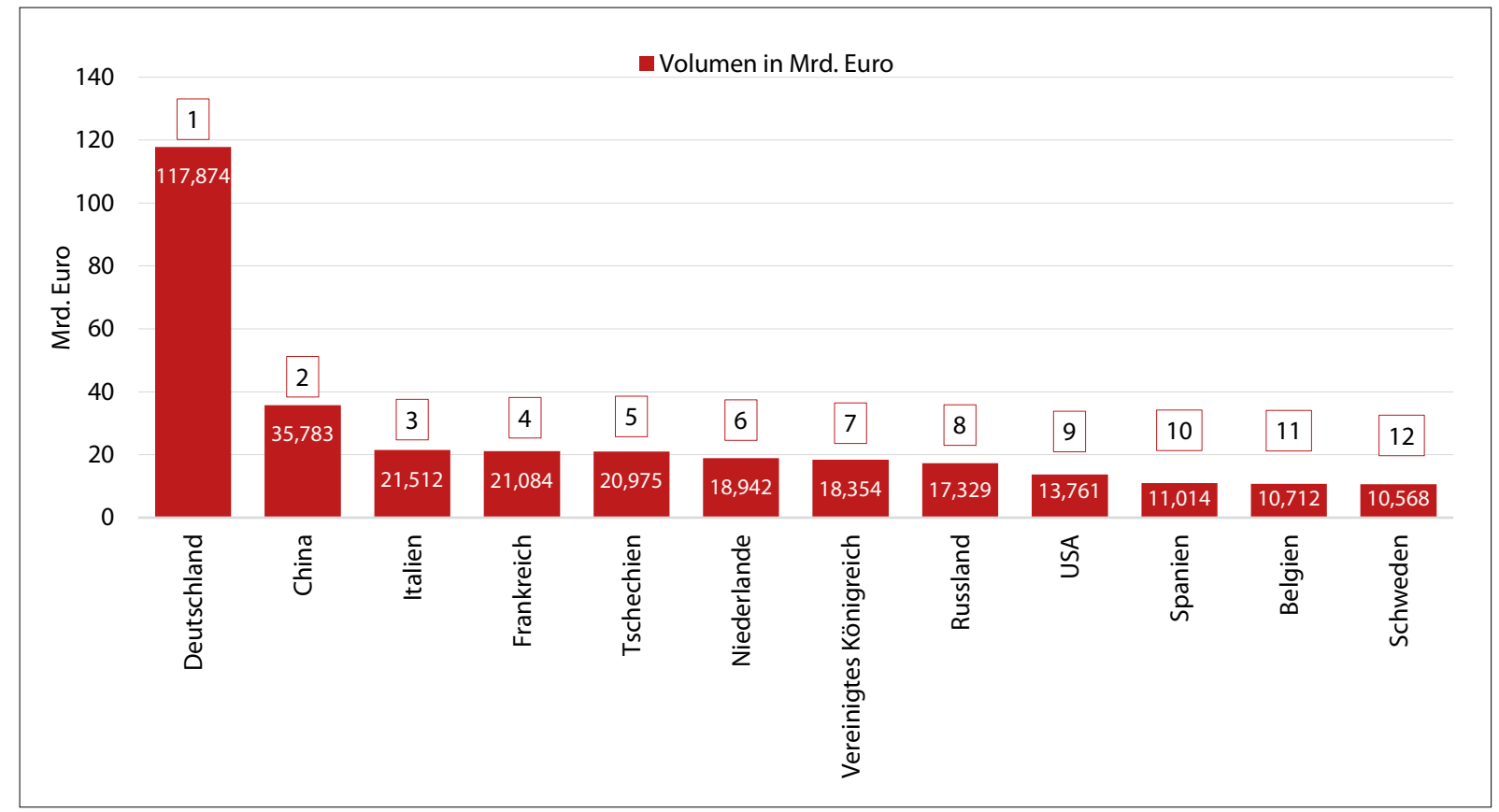

Quelle: Główny Urząd Statystyczny [Statistisches Hauptamt], Warschau; Berechnungen des Autors Adrian Stadnicki. 


\section{Jahrbuch Polen 2020 Polnische Wirtschaft}

Herausgegeben vom Deutschen Polen-Institut Darmstadt, Wiesbaden 2020, 200 S., zahlreiche Abb. Preis: $15 €$ (Abo 13,50 €), ISSN 1863-0278, ISBN 978-3-447-11404-2 www.deutsches-polen-institut.de Bestellung: verlag@harrassowitz.de oder kaluza@dpi-da.de

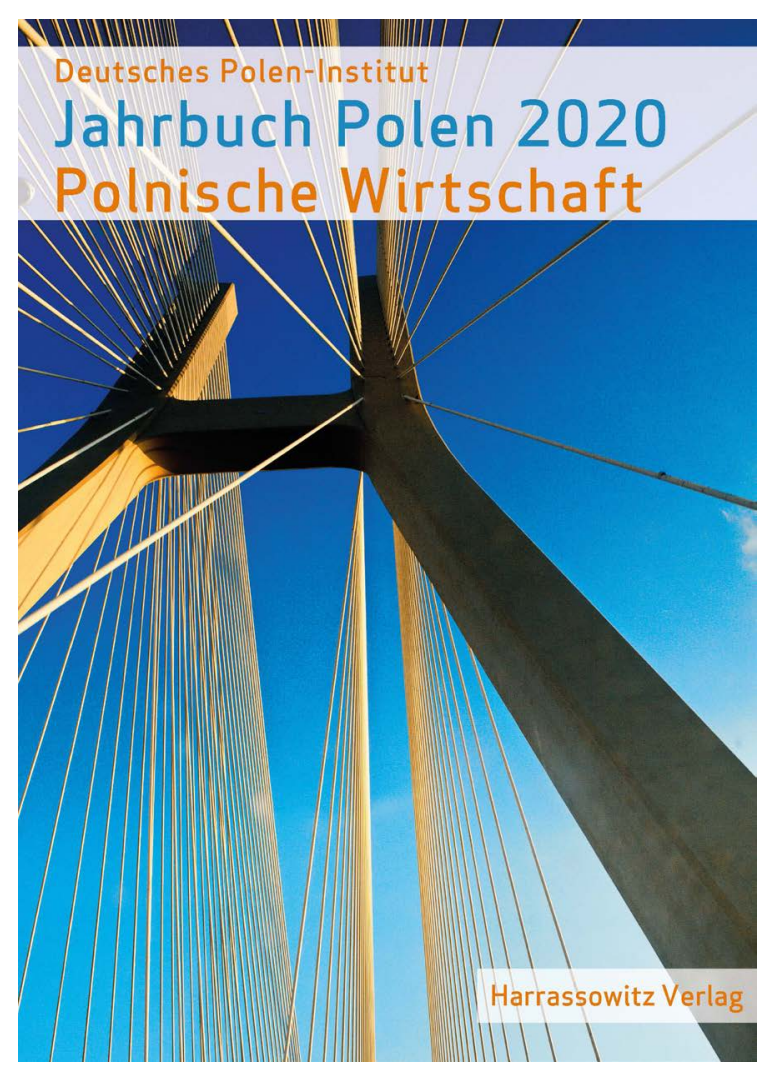

"Polnische Wirtschaft» war lange Zeit im deutschen Sprachgebrauch ein Ausdruck für Schlamperei, Unordnung, auch Rückständigkeit und Armut. Die Ursachen dafür waren vielfältig: die Schwäche der Städte, die Politik der Teilungsmächte, die Kriegsfolgen und die kommunistische Misswirtschaft. Noch vor dem EU-Beitritt des Landes 2004 zeigten westeuropäische Medien ein Pferdefuhrwerk auf dem Lande, das allgemein für die ökonomischen Verhältnisse in Polen wie für die gesamte ökonomische (Un)Ordnung in der osteuropäischen Peripherie stand. Und dennoch...

Unter hohen sozialen Kosten, in einer Zeit der politischen Veränderungen nach 1989, geschah ein kleines Wunder an der Weichsel - das Wachstum kehrte ein! Das Wachstum, das seit 1992 ununterbrochen fortdauert und Polen heute - nach mehr als 30 Jahren - zu einem der wichtigsten europäischen Wirtschaftsstandorte aufsteigen ließ.

Heute steht Polen vor neuen Herausforderungen - und es steht viel auf dem Spiel, denn "Made in Poland« ist zu einer Marke geworden. Dank einem enormen Qualitäts- und Technologieschub war das Land imstande, zu den größten Gewinnern der Globalisierung zu werden: Polens Exportquote liegt heute höher als die des deutschen Nachbarn. Beide Länder sind heute in Wahrheit ein gemeinsamer Wirtschaftsraum. Über Vorzüge und Nachteile dieser Beziehung, sowie über zahlreiche weitere, nicht nur strikt wirtschaftliche Themen, berichtet das aktuelle Jahrbuch Polen 2020 Polnische Wirtschaft.

Unsere Autoren schreiben u. a. über die deutsch-polnischen Wirtschaftsbeziehungen (Sebastian Płóciennik), Polens erfolgreiche Rolle in der Globalisierung (Grzegorz Siemiończyk), die regionalen Unterschiede (Grzegorz Masik), aber auch Probleme wie ausbleibende Innovationen (Edwin Bendyk), die problematische demografische Entwicklung (Bogusław Chrabota) und die notwendige Immigration (Piotr Arak). Dass Polen zum Musterknaben der europäischen Wirtschaft wird, war lange Zeit nicht absehbar, die Situation am Anfang der Transformation beleuchten deswegen u. a. Reinhold Vetter, Jakub Szumski und Leszek Balcerowicz. Schließlich beschäftigt sich Jan Sowa mit dem sozialen und psychologischen Preis der polnischen Konsumgesellschaft, während Karol Modzelewski nach dem politischen Preis der Einführung des Kapitalismus in Polen gefragt wird. Und Kacper Pobłocki und Krzysztof Jasiecki geben Einsichten in das Leben polnischer (Geld-)Eliten.

Inhalt

Einführung

Polnische Wirtschaft hat einen Ruf zu verlieren

Sebastian Płóciennik Besser geht's nicht? Die deutsch-polnischen Wirtschaftsbeziehungen

Grzegorz Siemiończyk Exportland Polen - Segen und Risiken der Globalisierung

Bogusław Chrabota

Edwin Bendyk

Polen auf dem Weg ins Jahr 2050

Vom Einholen und Überholen - Wo bleibt die Innovation?

Grzegorz Masik

Piotr Arak Gründe für regionales Wachstum Mehr als nur EU-Subventionen? Polen - Vom Auswanderungsland zum Einwanderungsland

Jan Sowa

Karol Modzelewski / Neue Ellenbogengesellschaft. Politische Genese und soziale Folgen des polnischen Kapitalismus

Grzegorz Sroczyński

Kacper Pobłocki

Krzysztof Jasiecki /

Grzegorz Sroczyński

Jakub Szumski

Sie sind sauer? Wunderbar!

Die transnationale Klasse im heutigen Polen

Woran denken polnische

Millionäre?

Schlangestehen. Traum und Wirklichkeit der sozialistischen Konsumgesellschaft

Reinhold Vetter Transformation als Eliteprojekt Debatten über Wirtschaftsreformen in den 1980er Jahren

Leszek Balcerowicz / Man muss kämpfen ...

Maria Stremecka

Jan Opielka

Ein Vergleich, kein Vergleich - Die transformierte Ökonomie in Polen und Tschechien 


\section{Covid-19 in Polen (Stand: 12. April 2021)}

Grafik 1: Die Ausbreitung von Covid-19 in Polen

(tägliche Fallzahlen, 1. März 2020 - 12. April 2021, Johns-Hopkins-Universität)

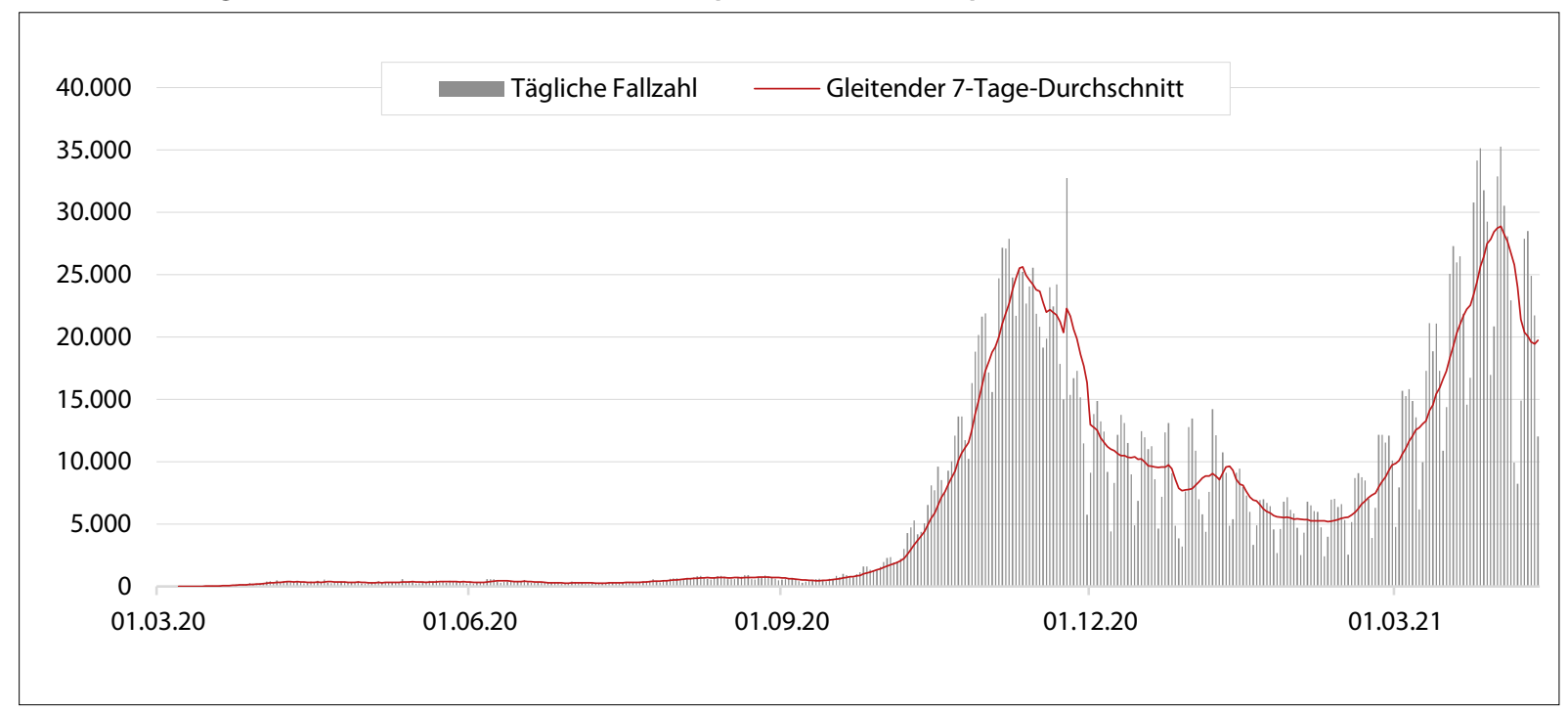

Quelle: Johns-Hopkins-Universität. Stand: 12.04.2021, 22:20 Uhr MESZ https://coronavirus.jhu.edu/map.html; https://github.com/CSSEGISandData/COVID-19/ blob/master/csse_covid_19_data/csse_covid_19_time_series/time_series_covid19_confirmed_global.csv

Tabelle 1: Die Ausbreitung von Covid-19 in Polen (16. März - 12. April 2021, Johns-Hopkins-Universität)

\begin{tabular}{|c|c|c|}
\hline Datum & Gesamtzahl der Fälle & Tägliche Fallzahl \\
\hline 16.03 .21 & 1.931 .921 & 14.394 \\
\hline 17.03 .21 & 1.956 .974 & 25.053 \\
\hline 18.03 .21 & 1.984 .248 & 27.274 \\
\hline 19.03 .21 & 2.010 .244 & 25.996 \\
\hline 20.03 .21 & 2.036 .700 & 26.456 \\
\hline 21.03 .21 & 2.058 .550 & 21.850 \\
\hline 22.03 .21 & 2.073 .129 & 14.579 \\
\hline 23.03 .21 & 2.089 .869 & 16.740 \\
\hline 24.03 .21 & 2.120 .671 & 30.802 \\
\hline 25.03 .21 & 2.154 .821 & 34.150 \\
\hline 26.03 .21 & 2.189 .966 & 35.145 \\
\hline 27.03 .21 & 2.221 .725 & 31.759 \\
\hline 28.03 .21 & 2.250 .991 & 29.266 \\
\hline 29.03 .21 & 2.267 .964 & 16.973 \\
\hline
\end{tabular}

\begin{tabular}{|c|c|c|}
\hline Datum & Gesamtzahl der Fälle & Tägliche Fallzahl \\
\hline 30.03 .21 & 2.288 .826 & 20.862 \\
\hline 31.03 .21 & 2.321 .717 & 32.891 \\
\hline 01.04 .21 & 2.356 .970 & 35.253 \\
\hline 02.04 .21 & 2.387 .511 & 30.541 \\
03.04 .21 & 2.415 .584 & 28.073 \\
\hline 04.04 .21 & 2.438 .542 & 22.958 \\
05.04 .21 & 2.448 .463 & 9.921 \\
\hline 06.04 .21 & 2.456 .709 & 8.246 \\
\hline 07.04 .21 & 2.471 .617 & 14.908 \\
\hline 08.04 .21 & 2.499 .507 & 27.890 \\
\hline 09.04 .21 & 2.528 .006 & 28.499 \\
\hline 10.04 .21 & 2.552 .898 & 24.892 \\
\hline 11.04 .21 & 2.574 .631 & 21.733 \\
\hline 12.04 .21 & 2.586 .647 & 12.016 \\
\hline
\end{tabular}

Für die Zahlen vom 01.03.2020-15.03.2021 siehe die Statistik »Covid-19 in Polen« in den Polen-Analysen 255 -271.

Quelle: Johns-Hopkins-Universität. Stand: 12.04.2021, 22:20 Uhr MESZ https://coronavirus.jhu.edu/map.html; https://github.com/CSSEGISandData/COVID-19/ blob/master/csse_covid_19_data/csse_covid_19_time_series/time_series_covid19_confirmed_global.csv 
Grafik 2: Todesfälle durch Covid-19 in Polen

(tägliche Todesfälle, 1. März 2020 - 12. April 2021, Johns-Hopkins-Universität)

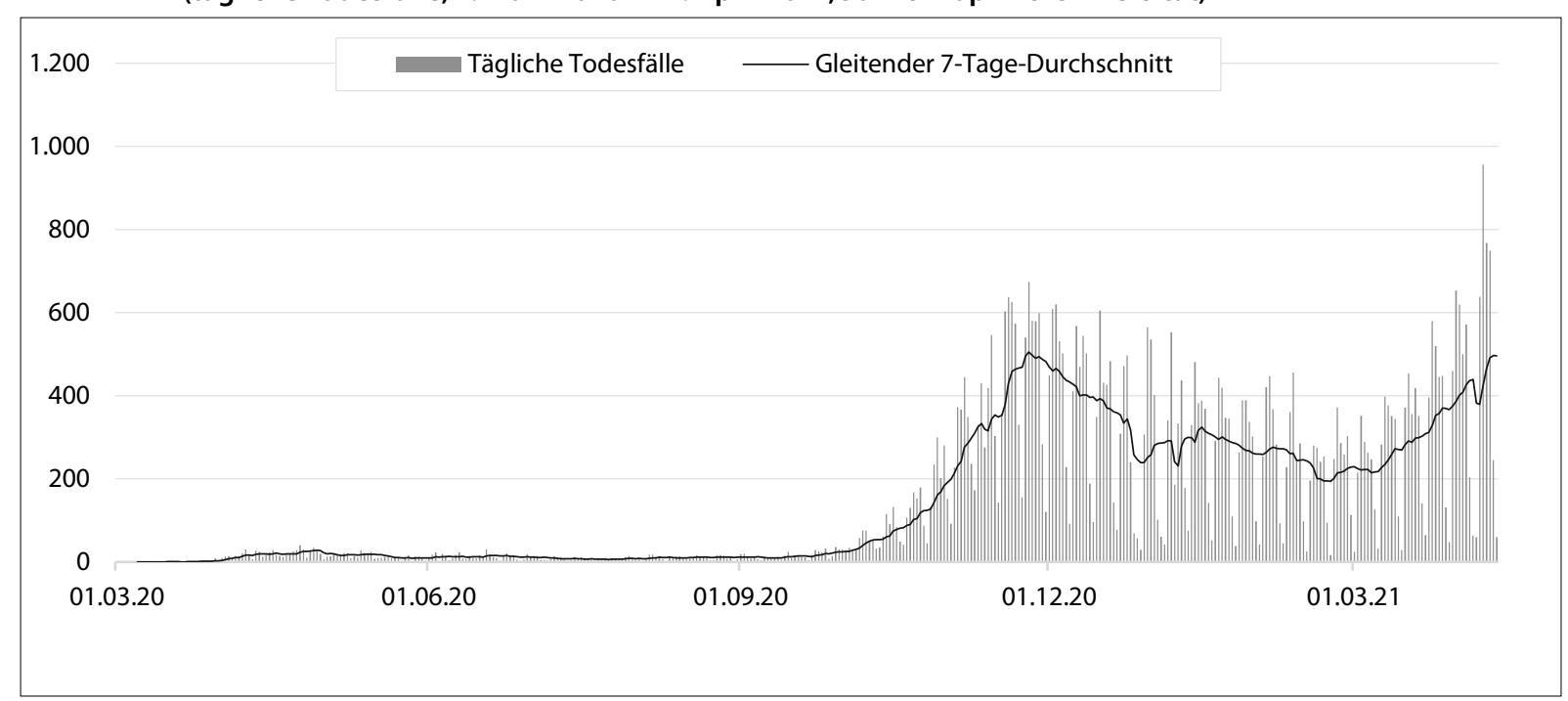

Quelle: Johns-Hopkins-Universität. Stand: 12.04.2021, 22:20 UhrMESZ https://coronavirus.jhu.edu/map.html; https://github.com/CSSEGISandData/COVID-19/ blob/master/csse_covid_19_data/csse_covid_19_time_series/time_series_covid19_deaths_global.csv

Tabelle 2: Todesfälle durch Covid-19 in Polen (16. März - 12. April 2021, Johns-Hopkins-Universität)

\begin{tabular}{|c|c|c|}
\hline Datum & $\begin{array}{c}\text { Gesamtzahl der } \\
\text { Todesfälle }\end{array}$ & Tägliche Todesfälle \\
\hline 16.03 .21 & 47.578 & 372 \\
\hline 17.03 .21 & 48.032 & 454 \\
\hline 18.03 .21 & 48.388 & 356 \\
\hline 19.03 .21 & 48.807 & 419 \\
\hline 20.03 .21 & 49.159 & 352 \\
\hline 21.03 .21 & 49.300 & 141 \\
\hline 22.03 .21 & 49.365 & 65 \\
\hline 23.03 .21 & 49.761 & 396 \\
\hline 24.03 .21 & 50.340 & 579 \\
\hline 25.03 .21 & 50.860 & 520 \\
\hline 26.03 .21 & 51.305 & 445 \\
\hline 27.03 .21 & 51.753 & 448 \\
\hline 28.03 .21 & 51.884 & 131 \\
\hline 29.03 .21 & 51.932 & 48 \\
\hline
\end{tabular}

\begin{tabular}{|c|c|c|}
\hline Datum & $\begin{array}{c}\text { Gesamtzahl der } \\
\text { Todesfälle }\end{array}$ & Tägliche Todesfälle \\
\hline 30.03 .21 & 52.392 & 460 \\
\hline 31.03 .21 & 53.045 & 653 \\
01.04 .21 & 53.665 & 620 \\
\hline 02.04 .21 & 54.165 & 500 \\
\hline 03.04 .21 & 54.737 & 572 \\
\hline 04.04 .21 & 54.941 & 204 \\
\hline 05.04 .21 & 55.005 & 64 \\
\hline 06.04 .21 & 55.065 & 60 \\
\hline 07.04 .21 & 55.703 & 638 \\
\hline 08.04 .21 & 56.659 & 956 \\
\hline 09.04 .21 & 57.427 & 768 \\
\hline 10.04 .21 & 58.176 & 749 \\
\hline 11.04 .21 & 58.421 & 245 \\
\hline 12.04 .21 & 58.481 & 60 \\
\hline
\end{tabular}

Für die Zahlen vom 01.03.2020-15.03.2021 siehe die Statistik »Covid-19 in Polen« in den Polen-Analysen 255 -271.

Quelle: Johns-Hopkins-Universität. Stand: 12.04.2021, 22:20 UhrMESZ https://coronavirus.jhu.edu/map.html; https://github.com/CSSEGISandData/COVID-19/ blob/master/csse_covid_19_data/csse_covid_19_time_series/time_series_covid19_deaths_global.csv 
Grafik 3: Covid-19-Infektionsfälle nach Woiwodschaften (12. April 2021, 10.30 Uhr)

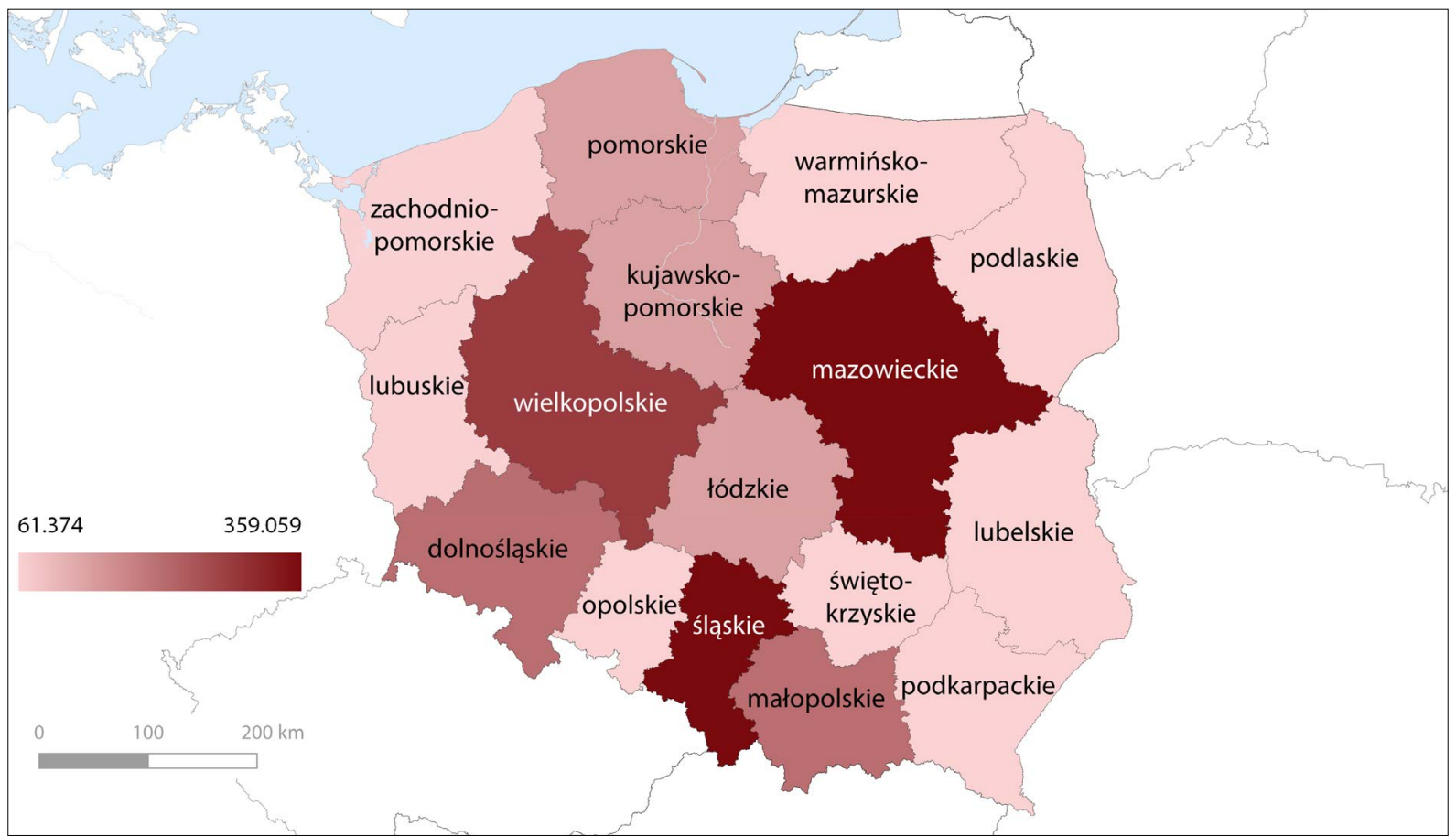

Quelle: Ministerstwo Zdrowia [Gesundheitsministerium], TVN24.pl. https://tvn24.pl/polska/koronawirus-w-polsce-mapa-zakazen-dzisiaj-ile-osob-zostalozaszczepionych-12-kwietnia-2021-4344739 (abgerufen am 12.04.2021); Karte erstellt von der Forschungsstelle Osteuropa an der Universität Bremen in QGIS mit Geodaten von https://www.openstreetmap.org.

\section{Grafik 4: Todesfälle durch Covid-19 nach Woiwodschaften (12. April 2021, 10.30 Uhr)}

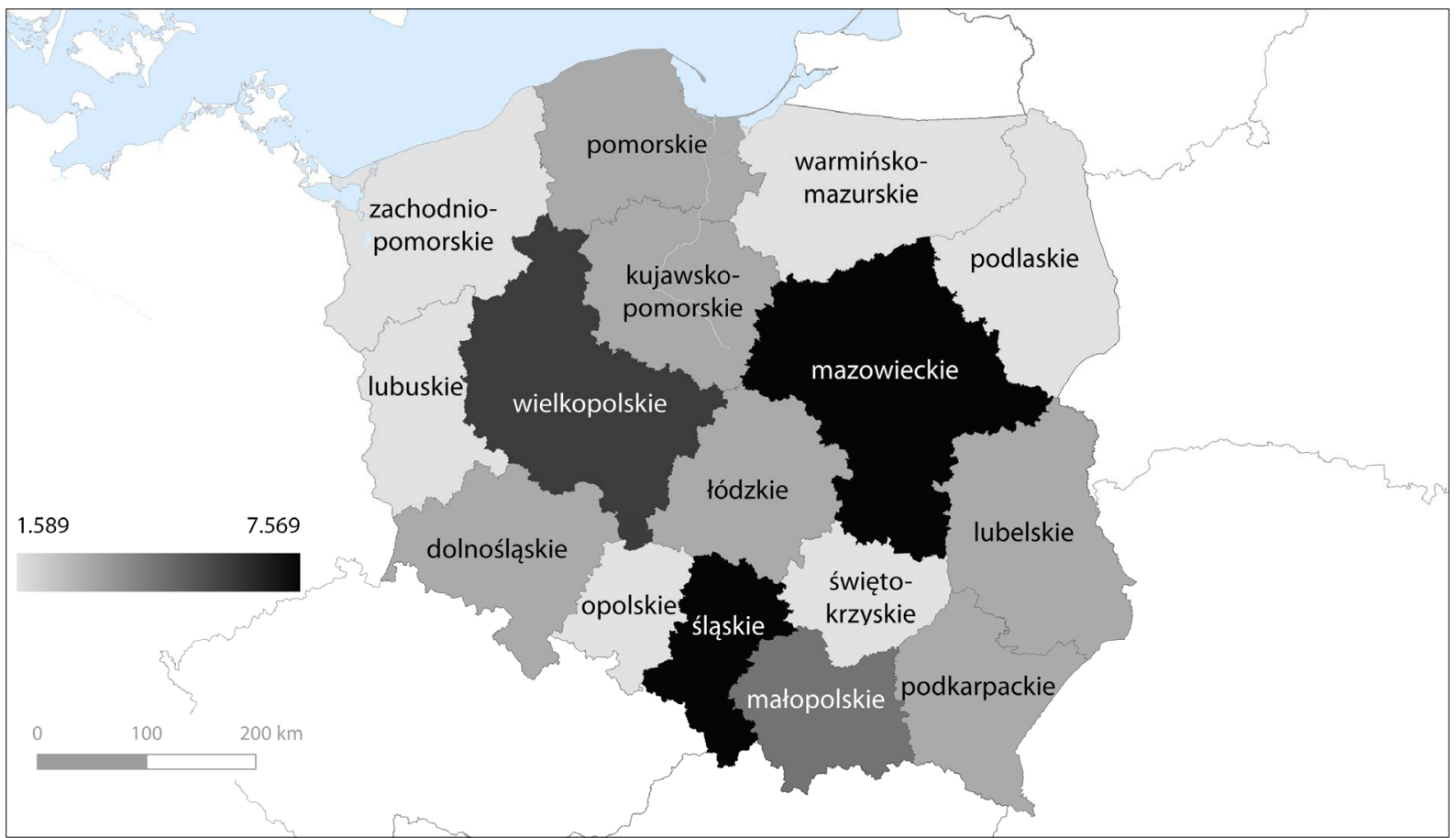

Quelle: Ministerstwo Zdrowia [Gesundheitsministerium], TVN24.pl. https://tvn24.pl/polska/koronawirus-w-polsce-mapa-zakazen-dzisiaj-ile-osob-zostalozaszczepionych-12-kwietnia-2021-4344739 (abgerufen am 12.04.2021); Karte erstellt von der Forschungsstelle Osteuropa an der Universität Bremen in QGIS mit Geodaten von https://www.openstreetmap.org. 
Grafik 5: Covid-19-Infektions- und Todesfälle nach Woiwodschaften im Vergleich (12. April 2021, 10.30 Uhr)

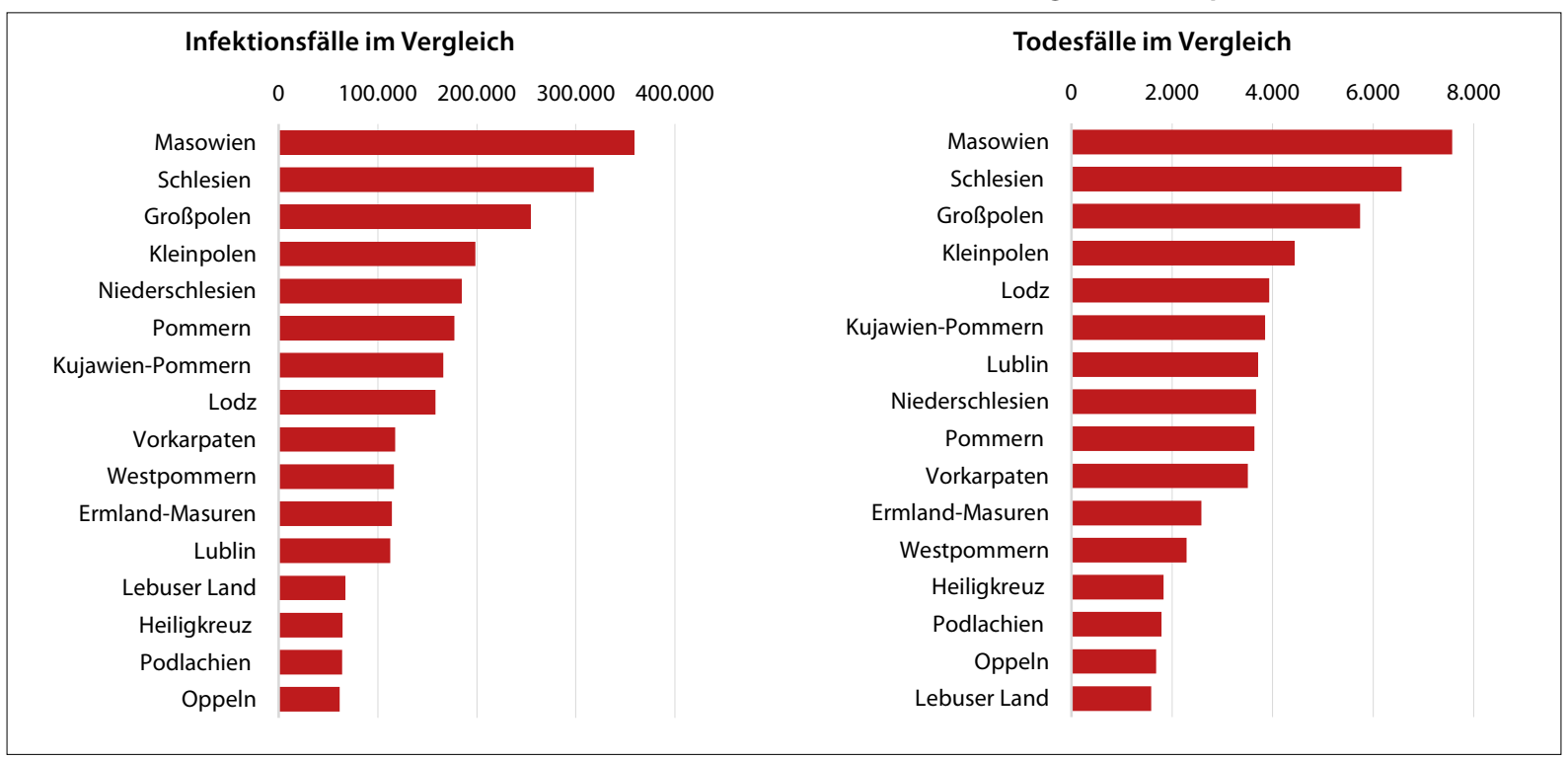

Quelle: Ministerstwo Zdrowia [Gesundheitsministerium], TVN24.pl. https://tvn24.pl/polska/koronawirus-w-polsce-mapa-zakazen-dzisiaj-ile-osob-zostalozaszczepionych-12-kwietnia-2021-4344739 (abgerufen am 12.04.2021)

Tabelle 3: Covid-19-Infektions- und Todesfälle nach Woiwodschaften im Vergleich (12. April 2021, 10.30 Uhr)

\begin{tabular}{|c|c|c|}
\hline Woiwodschaft (województwo) & Infektionsfälle insgesamt & Todesfälle insgesamt \\
\hline Ermland-Masuren (warmińsko-mazurskie) & 114.423 & 2.586 \\
\hline Großpolen (wielkopolskie) & 254.507 & 5.740 \\
\hline Heiligkreuz (świętokrzyskie) & 64.663 & 1.830 \\
\hline Kleinpolen (małopolskie) & 198.696 & 4.442 \\
\hline $\begin{array}{l}\text { Kujawien-Pommern } \\
\text { (kujawsko-pomorskie) }\end{array}$ & 166.335 & 3.853 \\
\hline Lebuser Land (lubuskie) & 67.482 & 1.589 \\
\hline Lodz (łódzkie) & 158.226 & 3.931 \\
\hline Lublin (lubelskie) & 112.648 & 3.715 \\
\hline Masowien (mazowieckie) & 359.059 & 7.569 \\
\hline Niederschlesien (dolnośląskie) & 184.796 & 3.669 \\
\hline Oppeln (opolskie) & 61.374 & 1.682 \\
\hline Podlachien (podlaskie) & 64.134 & 1.794 \\
\hline Pommern (pomorskie) & 177.373 & 3.638 \\
\hline Schlesien (śląskie) & 318.188 & 6.566 \\
\hline Vorkarpaten (podkarpackie) & 117.474 & 3.505 \\
\hline Westpommern (zachodniopomorskie) & 116.284 & 2.291 \\
\hline
\end{tabular}

Quelle: Ministerstwo Zdrowia [Gesundheitsministerium], TVN24.pl. https://tvn24.pl/polska/koronawirus-w-polsce-mapa-zakazen-dzisiaj-ile-osob-zostalozaszczepionych-12-kwietnia-2021-4344739 (abgerufen am 12.04.2021) 


\section{Covid-19-Chronik, 16. März - 12. April 2021}

\begin{tabular}{|c|c|}
\hline 16.03 .2021 & $\begin{array}{l}\text { Andrzej Gut-Mostowy, Vizeminister für Entwicklung, Arbeit und Technologie, teilt im Parlamentsausschuss } \\
\text { für Körperkultur, Sport und Tourismus mit, dass infolge der Corona-Pandemie im Jahr } 2020 \text { in der Tourismus- } \\
\text { branche ein Rückgang der inländischen Übernachtungsgäste von } 45 \% \text { verzeichnet wurde. Bei den ausländi- } \\
\text { schen Touristen lag die Anzahl um knapp } 70 \% \text { niedriger als im Vorjahr. Rafał Szlachta, Vorsitzender der Polni- } \\
\text { schen Tourismusorganisation (Polska Organizacja Turystyczna - POT), kündigt an, dass die POT im laufenden } \\
\text { Jahr verstärkt um ausländische Touristen werben wird, da sie } 70 \% \text { der Einnahmen für die Branche ausmachen. }\end{array}$ \\
\hline 16.03 .2021 & $\begin{array}{l}\text { In einer Stellungnahme wendet sich der Hauptärzterat (Naczelna Rada Lekarska) entschieden gegen einen Geset- } \\
\text { zesentwurf, der vorsieht, dass nicht nur Ärzte, sondern auch Vertreter anderer medizinischer Berufe Corona- } \\
\text { Impfungen durchführen dürfen. Hintergrund ist der Gesetzesentwurf der Fraktion von Recht und Gerechtig- } \\
\text { keit (Prawo i Sprawiedliwość - PiS), der aktuell von den Parteien diskutiert wird. }\end{array}$ \\
\hline 17.03 .2021 & $\begin{array}{l}\text { Gesundheitsminister Adam Niedzielski kündigt einen Lockdown für ganz Polen ab dem 20. März an. Der } \\
\text { Grund ist die dritte Corona-Infektionswelle, in der die britische Mutante des Corona-Virus für eine rasche } \\
\text { Verbreitung sorgt. In der vergangenen Woche habe sich die Zahl der Corona-Infizierten um } 38 \% \text { und die der } \\
\text { Todesfälle um } 24 \% \text { erhöht. Die Zahl der Corona-Patienten in Krankenhäusern sei um } 17 \% \text { gestiegen, } 74 \% \\
\text { der Beatmungsgeräte seien im Einsatz, so Niedzielski. Gemeinsam mit dem Innenminister habe er die Woi- } \\
\text { woden angewiesen, mehr Krankenhausbetten für Covid-19-Patienten bereitzuhalten. }\end{array}$ \\
\hline 18.03 .2021 & $\begin{array}{l}\text { In einem Interview von TVN } 24 \text { kritisiert Senatsmarschall Tomasz Grodzki (Bürgerkoalition/Koalicja Oby- } \\
\text { watelska-KO), dass das Gesundheitssystem bereits vor der Corona-Pandemie unzulänglich war. Darauf seien } \\
\text { die aktuell hohen Todeszahlen im Zusammenhang mit Covid-19 zurückzuführen. }\end{array}$ \\
\hline 20.03 .2021 & $\begin{array}{l}\text { Ab dem heutigen Tag gilt ein erneuter Lockdown in ganz Polen, zunächst bis zum 9. April. Hotels, Kulturein- } \\
\text { richtungen, Sporteinrichtungen und Geschäfte müssen schließen. Ausgenommen sind Lebensmittelgeschäfte, } \\
\text { Apotheken, Drogerien, Presse- sowie Buchläden, Zoohandlungen, Baumärkte, Banken, Friseure, Optiker und } \\
\text { Reinigungen. In den Schulen erhalten alle Klassen wieder Distanzunterricht. Empfohlen wird, soweit mög- } \\
\text { lich, die Arbeit im Homeoffice. }\end{array}$ \\
\hline 20.03 .2021 & $\begin{array}{l}\text { In Warschau protestieren einige Hundert Menschen unter dem Motto »Marsch der Freiheit« gegen die Ein- } \\
\text { schränkungen zur Bekämpfung der Corona-Pandemie. Es kommt zu Zusammenstößen mit der Polizei. }\end{array}$ \\
\hline 22.03 & $\begin{array}{l}\text { Der Impfbeauftragte der Regierung, Michał Dworczyk, teilt auf einer Pressekonferenz mit, dass die polni- } \\
\text { sche Regierung einer Bitte von NATO-Generalsekretär Jens Stoltenberg nachgekommen sei und } 20 \text { polnische } \\
\text { Ärzte in dieser Woche Impfungen gegen Covid-19 im NATO-Hauptquartier in Brüssel durchführen werden. } \\
\text { Dafür werden ca. 3.500 Impfdosen des Herstellers AstraZeneca (Großbritannien/Schweden) aus den polnischen } \\
\text { Beständen eingesetzt. Die Impfungen finden im Rahmen der Vorbereitungen des NATO-Gipfels im Juni statt. }\end{array}$ \\
\hline 23.03 & $\begin{array}{l}\text { Aktuellen Angaben der Regierung zufolge haben seit Beginn der Impfkampagne gegen Covid-19 am 27. Dezem- } \\
\text { ber } 2020 \text { bisher 3,29 Mio. Menschen in Polen die erste Impfung erhalten und 1,79 Mio. auch die zweite Impfung. }\end{array}$ \\
\hline 24.03 .2021 & $\begin{array}{l}\text { Angesichts der hohen Infektionszahlen der dritten Welle der Corona-Pandemie und der bevorstehenden Got- } \\
\text { tesdienste in der Karwoche und zu Ostern rufen Gesundheitsminister Adam Niedzielski und der Generalse- } \\
\text { kretär der Polnischen Bischofskonferenz, Bischof Artur Miziński, in einem gemeinsamen Appell die Priester } \\
\text { in den lokalen Kirchengemeinden auf, auf strenge Einhaltung der Abstands- und Hygieneregeln in den Got- } \\
\text { tesdiensten zu achten. }\end{array}$ \\
\hline 24.03 .2021 & $\begin{array}{l}\text { Landesweit beginnen die Corona-Impfungen der uniformierten Dienste. Auf einer Pressekonferenz teilt der } \\
\text { stellvertretende Innenminister Błażej Poboży mit, dass im Laufe der Corona-Pandemie in Polen bisher ein Fünf- } \\
\text { tel der Polizisten (ca. 20.000) an Covid-19 erkrankt ist. }\end{array}$ \\
\hline 25.03 .2021 & $\begin{array}{l}\text { Die Tageszeitung "Dziennik Gazeta Prawna« berichtet über die neuesten Angaben des Statistischen Haupt- } \\
\text { amtes (Główny Urząd Statystyczny - GUS) zur Lebenserwartung der Polen. Demnach sei die Lebenserwar- } \\
\text { tung bei den 60- bis 65-Jährigen infolge der Corona-Pandemie im Jahr } 2020 \text { um rund ein Jahr im Vergleich } \\
\text { zu } 2019 \text { gesunken. }\end{array}$ \\
\hline 26.03 .2021 & $\begin{array}{l}\text { In einem Interview der Tageszeitung "Dziennik Gazeta Prawna« stellt der Impfbeauftragte der Regierung, } \\
\text { Michał Dworczyk, in Aussicht, dass am Ende des dritten Quartals } 2021 \text { alle impfwilligen Polen eine Corona- } \\
\text { Impfung erhalten haben werden, da Polen bis Ende April ca. } 7 \text { Mio. und in den Folgemonaten noch mehr } \\
\text { Impfdosen erhalten soll. }\end{array}$ \\
\hline
\end{tabular}




\begin{tabular}{|c|c|}
\hline 27.03 .2021 & $\begin{array}{l}\text { Aufgrund steigender Corona-Infektionszahlen (»dritte Welle«) gelten ab dem heutigen Tag bis zunächst zum } \\
\text { 9. April } 2021 \text { neue zusätzliche Beschränkungen. Möbelhäuser und Baumärkte ab einer Fläche von } 2.000 \mathrm{~m}^{2} \\
\text { sowie Friseur- und Kosmetiksalons dürfen nicht mehr öffnen. In Geschäften mit einer Fläche bis } 100 \mathrm{~m}^{2} \text { darf } \\
\text { sich ein Kunde pro } 15 \mathrm{~m}^{2} \text { aufhalten, in größeren Geschäften eine Person pro } 20 \mathrm{~m}^{2} \text {. In Kirchen und anderen } \\
\text { Gotteshäusern ist eine Person pro } 20 \mathrm{~m}^{2} \text { zulässig. Krippen und Vorschulen nehmen nur noch die Kinder von } \\
\text { medizinischem Personal auf. Sporteinrichtungen dürfen nur noch von Berufssportlern genutzt werden. Emp- } \\
\text { fohlen wird, wenn möglich, im Homeoffice zu arbeiten. Für die bevorstehenden Ostertage wird dazu aufge- } \\
\text { rufen, zu Hause zu bleiben und die Kontakte auf die Angehörigen des eigenen Haushaltes zu beschränken. }\end{array}$ \\
\hline 30.03 .2021 & $\begin{array}{l}\text { Ab dem heutigen Tag gilt eine neue Verordnung für die Einreise nach Polen, ungeachtet des Verkehrsmittels. } \\
\text { Bei Einreise aus dem Schengenraum kann die verpflichtende Quarantäne durch einen negativen Corona-Test, } \\
\text { der nicht älter als } 48 \text { Stunden sein darf, aufgehoben werden. Einreisende aus anderen Ländern müssen sich in } \\
\text { Quarantäne begeben, auch wenn sie einen negativen Test vorlegen können. Für Personen, die gegen Covid-19 } \\
\text { geimpft sind, gilt die Quarantänepflicht nicht. Die Verordnung wurde vom Ministerrat beschlossen. }\end{array}$ \\
\hline 31.03 .2021 & $\begin{array}{l}\text { In einer Erklärung unterstreicht der Polnische Entwicklungsfonds (Polski Fundusz Rozwoju - PFR), dass er } \\
\text { Kontrollen der Auszahlungen aus dem Finanzschirm des PFR einleiten wird. Hintergrund ist die Berichterstat- } \\
\text { tung des öffentlichen Fernsehens TVP am Vortag, wonach Antragsteller beim PFR unrechtmäßig ca. } 200.000 \\
\text { Zloty erhalten haben sollen. Das Geld ist dafür bestimmt, Folgen der Corona-Pandemie abzumildern. }\end{array}$ \\
\hline 01.04 .2021 & $\begin{array}{l}\text { Ministerpräsident Mateusz Morawiecki kündigt die Bereitstellung von bis zu } 30 \text { Mrd. Zloty an, um Unter- } \\
\text { nehmen in der Corona-Pandemie zu unterstützen. Bisher habe die Regierung über } 200 \text { Mrd. Zloty dafür auf- } \\
\text { gewendet. Zudem soll die Liste der empfangsberechtigten Unternehmen erweitert werden. In Aussicht gestellt } \\
\text { werden u. a. Beihilfen für die Mietzahlungen. }\end{array}$ \\
\hline 02.04 .2021 & $\begin{array}{l}\text { In seiner in den sozialen Medien verbreiteten Ansprache am heutigen Karfreitag ruft Ministerpräsident Mateusz } \\
\text { Morawiecki die Polen dazu auf, angesichts der dritten Corona-Infektionswelle Ostern nur mit den Perso- } \\
\text { nen zu feiern, die zum eigenen Haushalt gehören. Polen befinde sich aktuell in der schwierigsten Phase der } \\
\text { Pandemiebekämpfung. }\end{array}$ \\
\hline 06.04 .2021 & $\begin{array}{l}\text { Die Abgeordneten der Fraktion Die Linke (Lewica), Agnieszka Dziemianowicz-Bąk und Małgorzata Prokop- } \\
\text { Paczkowska, begutachten in einer sogenannten Abgeordnetenkontrolle die Nationale Impfstrategie der Regie- } \\
\text { rung gegen Covid-19. Dazu führen sie Kontrollen in der Kanzlei des Vorsitzenden des Ministerrates durch, } \\
\text { deren Leiter der Impfbeauftragte der Regierung, Michał Dworczyk, ist. Auf der Pressekonferenz im Anschluss } \\
\text { kritisieren die Abgeordneten, dass der Krisenstab der Regierung Absprachen mündlich trifft und keine Proto- } \\
\text { kolle und Anwesenheitslisten geführt werden. Es gebe keine Dokumente, aus denen hervorgeht, wer für wel- } \\
\text { che Entscheidungen verantwortlich ist, so Dziemianowicz-Bąk. Prokop-Paczkowska ruft zur Transparenz bei } \\
\text { den Entscheidungen der Regierung auf. }\end{array}$ \\
\hline 07.04 .2021 & $\begin{array}{l}\text { Das Gesundheitsministerium teilt mit, dass die Einschränkungen zur Eindämmung der Corona-Pandemie } \\
\text { vom 27. März nicht wie bisher vorgesehen bis zum 9. April gelten, sondern bis zum 18. April } 2021 \text { verlängert } \\
\text { werden. Als Grund wird die angespannte Situation in den Krankenhäusern wegen der Behandlung von Covid- } \\
\text { 19-Patienten genannt. }\end{array}$ \\
\hline 07.04 .2021 & $\begin{array}{l}\text { Der Fraktionsvorsitzende von Die Linke (Lewica), Krzysztof Gawkowski, teilt auf einer Pressekonferenz mit, } \\
\text { dass die Fraktion beim Obersten Rechnungshof (Najwyższa Izba Kontroli - NIK) einen Antrag auf Kon- } \\
\text { trolle der Durchführung der Corona-Maßnahmen stellen wird. Insbesondere soll die Umsetzung der Natio- } \\
\text { nalen Impfstrategie gegen Covid-19 überprüft werden. Am Vortag hatten die Fraktionsmitglieder Agnieszka } \\
\text { Dziemianowicz-Bąk und Małgorzata Prokop-Paczkowska Intransparenz bei Entscheidungen der Regierung } \\
\text { und eine fehlende Dokumentation bemängelt. }\end{array}$ \\
\hline 08.04 .2021 & $\begin{array}{l}\text { Ministerpräsident Mateusz Morawiecki kündigt einen Ausbau der Anzahl der Corona-Impfzentren an, damit } \\
\text { jeder Bürger in der Nähe seines Wohnortes geimpft werden kann. Dafür sollen zum Beispiel Sporthallen, Schul- } \\
\text { zentren und Feuerwehrwachen genutzt werden. Der Impfbeauftragte der Regierung, Michał Dworczyk, kün- } \\
\text { digt für die kommende Woche die Inbetriebnahme eines »Impf-Drive-Through" als Pilotprojekt an. }\end{array}$ \\
\hline 09.04 .2021 & $\begin{array}{l}\text { Ab dem heutigen Tag dürfen laut Anordnung des Gesundheitsministeriums abgesehen von Ärzten auch Zahn- } \\
\text { ärzte, Krankenschwestern, Hebammen, Arzthelferinnen, Rettungshelfer und Schulkrankenschwestern Imp- } \\
\text { fungen gegen Covid-19 durchführen. Nach Absolvierung einer Schulung dürfen auch Studierende der Medi- } \\
\text { zin und der Krankenpflege im fortgeschrittenen Studium sowie Physiotherapeuten, Pharmazeuten und Labor- } \\
\text { angestellte impfen. }\end{array}$ \\
\hline
\end{tabular}




\begin{tabular}{|l|l|}
\hline 11.04.2021 & $\begin{array}{l}\text { Die Fraktionsabgeordneten der Bürgerkoalition (Koalicja Obywatelska - KO), Michał Szczerba und Dariusz } \\
\text { Joński, kündigen eine Anzeige bei der Obersten Kontrollkammer (Najwyższa Izba Kontroli - NIK) an. Hin- } \\
\text { tergrund ist der Vertrag zwischen dem Gesundheitsministerium und einem Waffenhändler aus Lublin vom } \\
\text { April 2020 über den Kauf von Beatmungsgeräten für Covid-19-Patienten. Auf den ausgestellten Rechnun- } \\
\text { gen sei keine Mehrwertsteuer angegeben, es hätten aber ca. 13 Mio. Zloty abgeführt werden müssen, so die } \\
\text { KO-Abgeordneten. }\end{array}$ \\
\hline 12.04 .2021 & $\begin{array}{l}\text { Ab dem heutigen Tag können sich 59-Jährige (Geburtsjahr 1962) einen Termin für eine Corona-Impfung } \\
\text { geben lassen. }\end{array}$ \\
\hline
\end{tabular}

\section{März - 12. April 2020}

\begin{tabular}{|c|c|}
\hline 2021 & $\begin{array}{l}\text { Daniel Obajtek, Vorstandsvorsitzender des mehrheitlich vom Staat gehaltenen Polnischen Mineralölkonzerns } \\
\text { Orlen, teilt mit, dass er beim Zentralen Antikorruptionsbüro (Centralne Biuro Antykorupcyjne - CBA) eine } \\
\text { Überprüfung seiner finanziellen Angelegenheiten beantragt hat. Das CBA bestätigt, die Kontrolle aufzuneh- } \\
\text { men, und teilt mit, dass es bereits zweimal Obajteks Finanzen geprüft hat. Hintergrund sind Medienberichte } \\
\text { der letzten Wochen über Obajteks Eigentumsverhältnisse und mögliche Unregelmäßigkeiten beim Erwerb. }\end{array}$ \\
\hline 17.03 .2021 & $\begin{array}{l}\text { Die parlamentarische Untersuchungsgruppe zur Aufklärung der Vorwürfe an Daniel Obajtek, Vorstandsvorsit- } \\
\text { zender des mehrheitlich vom Staat gehaltenen Polnischen Mineralölkonzerns Orlen, nimmt ihre Arbeit auf. Der } \\
\text { Gruppe gehören Abgeordnete der Fraktionen Bürgerkoalition (Koalicja Obywatelska - KO), Die Linke (Lewica) } \\
\text { und Polnische Koalition - Polnische Bauernpartei (Koalicja Polska - PSL) an. Hintergrund sind Medienberichte } \\
\text { der letzten Wochen über Obajteks Eigentumsverhältnisse und mögliche Unregelmäßigkeiten beim Erwerb. }\end{array}$ \\
\hline 17.03.2021 & $\begin{array}{l}\text { Robert Biedroń, Parteivorsitzender von Frühling (Wiosna), und Włodzimierz Czarzasty, Parteichef der Demo- } \\
\text { kratischen Linksallianz (Sojusz Lewicy Demokratycznej-SLD), geben den offiziellen Zusammenschluss ihrer } \\
\text { Parteien unter dem Namen Neue Linke (Nowa Lewica) bekannt. Der neue Parteivorstand soll paritätisch } \\
\text { besetzt werden. Der Parteitag, auf dem der Vorstand gewählt und das Programm verabschiedet werden soll, } \\
\text { soll im Sommer stattfinden. }\end{array}$ \\
\hline 17.03.2021 & $\begin{array}{l}\text { Im Sejm findet die erste Lesung des von Bürgern eingebrachten Gesetzesentwurfes »Ja zur Familie, nein zu Gen- } \\
\text { der« statt. Der Entwurf wurde u. a. vom rechtskonservativen Rechtsinstitut Ordo Iuris vorbereitet; befürwor- } \\
\text { tet wird die Aufkündigung der sog. Istanbul-Konvention des Europarats („Übereinkommen des Europarats zur } \\
\text { Verhütung und Bekämpfung von Gewalt gegen Frauen und häuslicher Gewalt«) vonseiten Polens. Vor dem Sejm } \\
\text { wird gegen den Gesetzesentwurf protestiert; dazu hatte das Netzwerk Landesweiter Frauenstreik (Ogólnopolski } \\
\text { Strajk Kobiet) aufgerufen. Vorher kam es zu einer Gegendemonstration der Allpolnischen Jugend (Młodzież } \\
\text { Wszechpolska) am Denkmal der Heimatarmee (Armia Krajowa) und des polnischen Untergrundstaates. }\end{array}$ \\
\hline 18.03 .2021 & $\begin{array}{l}\text { Leszek Miller, ehemaliger Parteivorsitzender der Demokratischen Linksallianz (Sojusz Lewicy Demokratycznej- } \\
\text { SLD), kritisiert in einer in den sozialen Medien verbreiteten Stellungnahme, dass der Zusammenschluss der } \\
\text { SLD mit der Partei Frühling (Wiosna) nicht mit den Mitgliedern konsultiert wurde und es kein Referendum } \\
\text { dazu gab. Er werde sich nicht in eine andere Partei umschreiben lassen und fordert die SLD-Mitglieder auf, } \\
\text { selbständige Entscheidungen in dieser Angelegenheit zu treffen. Der Parteienzusammenschluss wurde am } \\
\text { Vortag von den Parteichefs Włodzimierz Czarzasty (SLD) und Robert Biedroń (Frühling) bekannt gegeben. }\end{array}$ \\
\hline 19.03 .2021 & $\begin{array}{l}\text { Ryszard Czarnecki, Europaabgeordneter der Europäischen Konservativen und Reformer aus den Reihen von } \\
\text { Recht und Gerechtigkeit (Prawo i Sprawiedliwość - PiS), sagt der Radionachrichtenagentur (Informacyjna } \\
\text { Agencja Radiowa), dass die PiS-Parteiführung über eine eventuelle Zusammenarbeit mit den nationalistischen } \\
\text { Parteien Fidesz (Ungarn) und Lega Nord (Italien) im Europäischen Parlament entscheiden wird. Die Zusam- } \\
\text { menarbeit mit Parteien, welche die Idee des Europas der Vaterländer vertreten, sei notwendig, so Czarnecki. } \\
\text { Fidesz hat am Vortag formal die Europäische Volkspartei (EVP) verlassen; die Lega Nord gehört der Fraktion } \\
\text { Identität und Demokratie im Europäischen Parlament an. }\end{array}$ \\
\hline
\end{tabular}




\begin{tabular}{|c|c|}
\hline 21.03.2021 & $\begin{array}{l}\text { Die stellvertretende Fraktionsvorsitzende von Die Linke (Lewica), Monika Pawłowska, gibt ihren Austritt aus } \\
\text { der Fraktion sowie aus der Partei Frühling (Wiosna) und ihren Eintritt in die Partei Verständigung (Porozu- } \\
\text { mienie) bekannt. Sie tritt jedoch nicht in die Fraktion der Regierungskoalition ein. Als Grund für ihren Wech- } \\
\text { sel nannte sie, dass die Linke einst soziale Sensibilität ausgezeichnet habe, jetzt beschäftige sie sich aber vor } \\
\text { allem mit weltanschaulichen Debatten. }\end{array}$ \\
\hline 21.03.2021 & Der Lyriker, Schriftsteller und Essayist Adam Zagajewski stirbt im Alter von 75 Jahren in Krakau (Kraków). \\
\hline 22.03.2021 & $\begin{array}{l}\text { Außenminister Zbigniew Rau nimmt an der Sitzung des Rates »Auswärtige Angelegenheiten« der Europäi- } \\
\text { schen Union teil. In der Aussprache über Russland unterstreicht Rau die Notwendigkeit, dass die EU Repres- } \\
\text { sionen gegenüber der Zivilgesellschaft in Russland verurteilt. Die Einhaltung der Menschenrechte sollte eines } \\
\text { der Haupthemen des politischen Dialogs mit Russland sein. }\end{array}$ \\
\hline 22.03 .2021 & $\begin{array}{l}\text { Präsident Andrzej Duda verleiht Robert Lewandowski, Fußballspieler der Nationalmannschaft und des deut- } \\
\text { schen Vereins Bayern München, das Komturskreuz des Ordens der Wiedergeburt Polens für herausragende } \\
\text { sportliche Leistungen und Werbung für Polen auf internationaler Bühne. }\end{array}$ \\
\hline 23.03.2021 & $\begin{array}{l}\text { Das Außenministerium bestellt den Charges d'Affaires der Botschaft der Republik Belarus ein und protestiert } \\
\text { gegen die Festnahme von Andżelika Borys, Vorsitzende des Verbands der Polen in Belarus. Das Vorgehen der } \\
\text { belarussischen Behörden widerspreche den internationalen Verpflichtungen Belarus' für den Schutz von Min- } \\
\text { derheiten und den bilateralen Verpflichtungen zum Schutz der polnischen Minderheit in Belarus. Das Außen- } \\
\text { ministerium ruft Belarus auf, Borys schnellstmöglich freizulassen und die willkürliche Verfolgung von Vertre- } \\
\text { tern der polnischen Minderheit zu unterlassen. }\end{array}$ \\
\hline 24.03.2021 & $\begin{array}{l}\text { Ministerpräsident Mateusz Morawiecki kündigt an, dass er auf der Sitzung des Europarates in den kommen- } \\
\text { den zwei Tagen die Festnahme der Vorsitzenden des Verbands der Polen in Belarus, Andżelika Borys, und ihre } \\
\text { Inhaftierung thematisieren wird. Belarus verstoße gegen die internationalen Standards und Polen widerspre- } \\
\text { che dieser Art von Geiselnahme, unterstreicht Morawiecki. }\end{array}$ \\
\hline 25.03.2021 & $\begin{array}{l}\text { Michał Dworczyk, Chef der Kanzlei des Ministerpräsidenten, teilt mit, dass am selben Tag Andrzej Poczobut, } \\
\text { Vorstandsmitglied des Verbands der Polen in Belarus, in Grodno (Belarus) verhaftet wurde. }\end{array}$ \\
\hline 25.03 & $\begin{array}{l}\text { Beim online-Gipfel der EU-Staats- und Regierungschefs thematisiert Ministerpräsident Mateusz Morawiecki } \\
\text { die Festnahmen von Angehörigen der polnischen Minderheit in Belarus und die Ausweisung polnischer Diplo- } \\
\text { maten aus Belarus, die unlängst stattfanden. Er ruft dazu auf, rasch eine weitere Liste von Sanktionen aufzustel- } \\
\text { len sowie über den aktuellen Stand des Plans zur wirtschaftlichen Unterstützung von Belarus zu informieren. }\end{array}$ \\
\hline 27.03.2021 & $\begin{array}{l}\text { Zbigniew Ziobro, Parteivorsitzender von Solidarisches Polen (Solidarna Polska) und Justizminister, ruft die pol- } \\
\text { nische Politik dazu auf, die Ratifizierung der Vereinbarung des Europäischen Rates über die gemeinsame euro- } \\
\text { päische Kreditaufnahme am Kapitalmarkt zur Bewältigung der Folgen der Corona-Pandemie in Polen nicht } \\
\text { weiterzuverfolgen. Zunächst solle eine sachorientierte Debatte mit Experten geführt werden, welche Folgen die } \\
\text { im Dezember } 2020 \text { von den europäischen Staats- und Regierungschefs getroffene Vereinbarung für den pol- } \\
\text { nischen Staat und seine Wirtschaft hätte. }\end{array}$ \\
\hline 27.03.202 & $\begin{array}{l}\text { Präsidentenberater Andrzej Zybertowicz sagt in einem Fernsehinterview im Sender TVP 3, dass Präsident } \\
\text { Andrzej Duda die Verhaftung von Repräsentanten der polnischen Minderheit in Belarus auf internationa- } \\
\text { ler Ebene thematisiert. Er habe einen Brief an den US-amerikanischen Präsidenten Joe Biden geschrieben } \\
\text { und Gespräche mit dem Präsidenten von Estland, dem Generalsekretär der Organisation für Sicherheit und } \\
\text { Zusammenarbeit in Europa (OSZE), dem Hohen Vertreter der OSZE für nationale Minderheiten sowie dem } \\
\text { Botschafter der Republik Belarus geführt. Auf Seiten der Europäischen Union sei nicht der politische Wille } \\
\text { zu erkennen, auf den Machtmissbrauch Belarus' zu reagieren. Die EU befasse sich lieber mit frei erfundenen } \\
\text { Rechtsbrüchen auf Seiten Polens, so Zybertowicz. Hintergrund sind die Verhaftungen von Andżelika Borys } \\
\text { und Andrzej Poczobut, die zum Vorstand des Verbands der Polen in Belarus gehören, sowie weiterer Mitglie- } \\
\text { der in führenden Positionen in dieser Woche. }\end{array}$ \\
\hline 29.03.2021 & $\begin{array}{l}\text { Ministerpräsident Mateusz Morawiecki reicht beim Verfassungstribunal (Trybunał Konstytucyjny-TK) einen } \\
\text { Antrag auf Überprüfung ein, ob das Recht der Europäischen Union Vorrang vor der polnischen Verfassung hat. }\end{array}$ \\
\hline 30.03 .2021 & $\begin{array}{l}\text { Per Akklamation verabschiedet der Sejm einen Beschluss, in dem er die »brutalen Repressionen« verurteilt, die } \\
\text { die belarussische Regierung gegenüber Angehörigen der polnischen Minderheit in Belarus anwendet. Das Vor- } \\
\text { gehen der belarussischen Regierung stehe in offenkundigem Widerspruch zu den internationalen Standards } \\
\text { gegenüber nationalen Minderheiten und den bilateralen Vereinbarungen. Der Sejm fordert die unverzügliche } \\
\text { Freilassung der Angehörigen der polnischen Minderheit, die in dieser Woche und davor festgenommen wurden. }\end{array}$ \\
\hline
\end{tabular}




\begin{tabular}{|c|c|}
\hline 31.03 .2021 & $\begin{array}{l}\text { Die Europäische Kommission reicht beim Gerichtshof der Europäischen Union (EuGH) Beschwerde gegen die } \\
\text { Tätigkeiten der Disziplinarkammer am Obersten Gericht (Sąd Najwyższy - SN) ein und beantragt die Ver- } \\
\text { hängung vorläufiger Maßnahmen bis zur Urteilsverkündung. Die EU-Kommission sehe die Unabhängigkeit } \\
\text { von Richtern in Polen in Gefahr, so EU-Justizkommissar Didier Reynders. U. a. geht es um das sogenannte } \\
\text { Disziplinierungsgesetz, auf dessen Grundlage Richter entlassen, herabgestuft oder mit Geldstrafen belegt wer- } \\
\text { den können, wenn sie die Entscheidungskompetenz oder Legalität eines anderen Richters, einer Kammer oder } \\
\text { eines Gerichts infrage stellen. }\end{array}$ \\
\hline 01.04 .2021 & $\begin{array}{l}\text { Vom heutigen Tag bis zum 30. Juni } 2021 \text { wird eine Volkszählung in Polen durchgeführt. Sie findet alle zehn } \\
\text { Jahre statt, die Teilnahme ist verpflichtend. Erhoben werden demografische Daten, Angaben zu Bildung, wirt- } \\
\text { schaftlicher Aktivität, Migration, ethnisch-kulturellen Spezifika sowie zum Wohnbestand. Die Datenabfrage } \\
\text { findet online und in mündlicher Form statt. }\end{array}$ \\
\hline 01.04 .2021 & $\begin{array}{l}\text { In Budapest treffen sich Viktor Orbán, Ministerpräsident Ungarns und Parteivorsitzender von Fidesz, Mateusz } \\
\text { Morawiecki, Ministerpräsident Polens aus der Partei Recht und Gerechtigkeit (Prawo i Sprawiedliwość - PiS), } \\
\text { und Matteo Salvini, Parteivorsitzender der italienischen Lega Nord. Thematisiert wird u. a. die Zukunft der } \\
\text { rechten Parteien im Europäischen Parlament. }\end{array}$ \\
\hline 06.04 .2021 & $\begin{array}{l}\text { Regierungssprecher Piotr Müller zeigt sich in einem Fernsehinterview mit »Telewizja Republika« optimistisch, } \\
\text { dass im Europäischen Parlament ein neuer politischer Block auf Initiative der Partei Recht und Gerechtigkeit } \\
\text { (Prawo i Sprawiedliwość - PiS), der ungarischen Fidesz-Partei und der italienischen Lega Nord gebildet wer- } \\
\text { den kann. Nach dem Treffen in der vergangenen Woche seien weitere Treffen der Parteien zur Ausarbeitung } \\
\text { eines Programms vorgesehen. Grundlage des Programms sollen die Idee der EU als Gemeinschaft souverä- } \\
\text { ner Staaten sowie konservative Werte wie Familie, nationale Identität und die Freiheit des Individuums sein. }\end{array}$ \\
\hline 07.04.2021 & $\begin{array}{l}\text { Das Kreisgericht in Warschau hat die Partei Polen } 2050 \text { von Szymon Hołownia (Polska } 2050 \text { Szymona Hołowni) } \\
\text { registriert. Parteivorsitzender ist Michał Kobosko. }\end{array}$ \\
\hline 08.04 .2021 & $\begin{array}{l}\text { Außenminister Zbigniew Rau trifft sich in Kiew (Ukraine) u. a. mit seinem ukrainischen Amtskollegen Dmytro } \\
\text { Kuleba. Es herrsche Übereinstimmung in der Einschätzung, dass das deutsch-russische Gaspipeline-Projekt } \\
\text { Nord Stream } 2 \text { eine dauerhafte Bedrohung des Friedens in Europa darstelle, da es zur Bündelung von politi- } \\
\text { schem, wirtschaftlichem und militärischem Druck genutzt werden könne, heißt es in der Stellungnahme des } \\
\text { polnischen Außenministeriums. Mit Blick auf den russisch-ukrainischen Konflikt in der Ostukraine betont } \\
\text { Rau die territoriale Integrität der Ukraine. }\end{array}$ \\
\hline 09.04 .2021 & $\begin{array}{l}\text { Die Landesstaatsanwaltschaft teilt mit, dass die Untersuchung der Ursachen der Flugzeugkatastrophe von Smo- } \\
\text { lensk (2010) bis zum 31. Dezember } 2021 \text { verlängert wird. An den Untersuchungen sind internationale Exper- } \\
\text { ten beteiligt. Bisher habe Russland nicht auf die Mahnschreiben der Landesstaatsanwaltschaft auf Herausgabe } \\
\text { des Flugzeugwracks reagiert. Bei dem Flugzeugabsturz verunglückten } 96 \text { Personen des politischen und öffent- } \\
\text { lichen Lebens Polens, darunter Präsident Lech Kaczyński, tödlich. }\end{array}$ \\
\hline 12.04 .2021 & $\begin{array}{l}\text { Ministerpräsident Mateusz Morawiecki kündigt an, dass die Regierung in den kommenden Jahren mehr als } \\
30 \text { Mrd. Zloty für die Straßeninfrastruktur auf Orts-, Gemeinde- und Kreisebene zur Verfügen stellen wird. }\end{array}$ \\
\hline
\end{tabular}

Sie können die gesamte Chronik seit 2007 auch auf http://www.laender-analysen.de/polen/ unter dem Link "Chronik« lesen. 


\section{ÜBER DIE POLEN-ANALYSEN}

Die Polen-Analysen erscheinen zweimal monatlich als E-Mail-Dienst. Sie werden gemeinsam vom Deutschen PolenInstitut Darmstadt, der Forschungsstelle Osteuropa an der Universität Bremen und weiteren Partnern eines Konsortiums (siehe Titelseite) herausgegeben.

Ein Archiv der Polen-Analysen finden Sie im Internet unter www.laender-analysen.de/polen Kostenloses Abonnement unter http://www.deutsches-polen-institut.de/Newsletter/subscribe.php

Diese Analysen finden Sie online als Lizenzausgabe auf

bpb.de

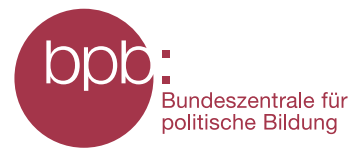

Deutsches Polen-Institut Darmstadt (www.deutsches-polen-institut.de)

Das seit 1980 tätige Deutsche Polen-Institut Darmstadt (DPI) ist ein Forschungs-, Informations- und Veranstaltungszentrum für polnische Kultur, Geschichte, Politik, Gesellschaft und die deutsch-polnischen Beziehungen, die sich im Kontext der europäischen Integration entwickeln. Institutionelle Träger des DPI sind das Land Hessen, die Kultusminister der Länder, das Auswärtige Amt und die Wissenschaftsstadt Darmstadt. Einen wesentlichen Beitrag zur Verwirklichung der Institutsziele leisten private Stiftungen. Ziel der Vermittlertätigkeit des DPI ist es, "die zu interessieren, auf die es politisch, wirtschaftlich, gesellschaftlich und kulturell im deutsch-polnischen Verhältnis ankommt (Leitlinien 1997). Es geht um die Entscheider und Multiplikatoren in Politik, Kultur, Bildung, Verwaltung, Medien und Wirtschaft. Das DPI versteht sich in Kooperation mit den Orten wissenschaftlicher Polen-Kompetenz an deutschen Hochschulen und Forschungsinstituten als verbindendes und vernetzendes Zentrum. Mit der 70.000 Bände zählenden multidisziplinären Fachbibliothek für Polen, die eine einzigartige Sammlung polnischer Belletristik in der Originalsprache und in deutscher Übersetzung umfasst, ist das DPI ein geschätzter Ort der Recherche und des wissenschaftlichen Arbeitens.

Forschungsstelle Osteuropa an der Universität Bremen (www.forschungsstelle.uni-bremen.de)

1982 gegründet, widmet sich die Forschungsstelle Osteuropa an der Universität Bremen der interdisziplinären Analyse der Länder Ost- und Ostmitteleuropas in Zeitgeschichte und Gegenwart. Der Forschungsschwerpunkt liegt dabei auf der Rolle von "Dissens und Konsens«, von Opposition und Zivilgesellschaft in ihrem historischen, politischen, gesellschaftlichen und kulturellen Kontext. Die Forschungsstelle besitzt in ihrem Archiv eine einzigartige Sammlung alternativer Kulturgüter und unabhängiger Texte aus den ehemaligen sozialistischen Ländern. Darunter befindet sich auch eine umfangreiche Sammlung des "Zweiten Umlaufs«, die das Schrifttum und Dokumente unabhängiger Initiativen und gesellschaftlicher Gruppen in Polen aus der Zeit von 1976 bis zum Umbruch umfasst. Hinzu kommt eine umfangreiche Bibliothek mit wissenschaftlicher Literatur. Mit Archiv, Bibliothek und zwei wissenschaftlichen Abteilungen ist die Forschungsstelle auch eine Anlaufstelle sowohl für Gastwissenschaftler als auch für die interessierte Öffentlichkeit.

Eine der Hauptaufgaben der Forschungsstelle ist die Information der interessierten Öffentlichkeit. Dazu gehören unter anderem regelmäßige E-Mail-Informationsdienste für Politik, Wirtschaft, Zivilgesellschaft und Medien.

Herausgeber:

Deutsches Polen-Institut, Deutsche Gesellschaft für Osteuropakunde e.V., Forschungsstelle Osteuropa an der Universität Bremen, Leibniz-Institut für Agrarentwicklung in Transformationsökonomien, Leibniz-Institut für Ost- und Südosteuropaforschung, Zentrum für Osteuropa- und internationale Studien (ZOiS) gGmbH

Redaktion:

Dr. Andrzej Kaluza (verantwortlich) (Darmstadt) und Silke Plate M.A. (Bremen)

Satz: Matthias Neumann

Wissenschaftlicher Beirat:

Prof. Dr. Stefan Garsztecki, Technische Universität Chemnitz

Prof. Dr. Klaus Ziemer, Kardinal-Stefan-Wyszyński-Universität Warschau

Die Meinungen, die in den Polen-Analysen geäußert werden, geben ausschließlich die Auffassung der Autoren wieder.

Abdruck und sonstige publizistische Nutzung sind nach Rücksprache mit der Redaktion gestattet. Polen-Analysen-Layout: Cengiz Kibaroglu, Matthias Neumann

Alle Ausgaben der Polen-Analysen sind mit Themen- und Autorenindex archiviert unter www.laender-analysen.de

ISSN 1863-9712 @ 2021 by Deutsches Polen-Institut, Deutsche Gesellschaft für Osteuropakunde e.V., Forschungsstelle Osteuropa an der Universität Bremen, Leibniz-Institut für Agrarentwicklung in Transformationsökonomien, Leibniz-Institut für Ost- und Südosteuropaforschung, Zentrum für Osteuropa- und internationale Studien (ZOiS) gGmbH

Kontakt: Dr. Andrzej Kaluza, Deutsches Polen-Institut, Residenzschloss, Marktplatz 15, 64283 Darmstadt,

Tel.: +49/6151/4202-20, Fax: +49/6151/4202-10, E-Mail: kaluza@dpi-da.de, Internet: www.laender-analysen.de/polen 


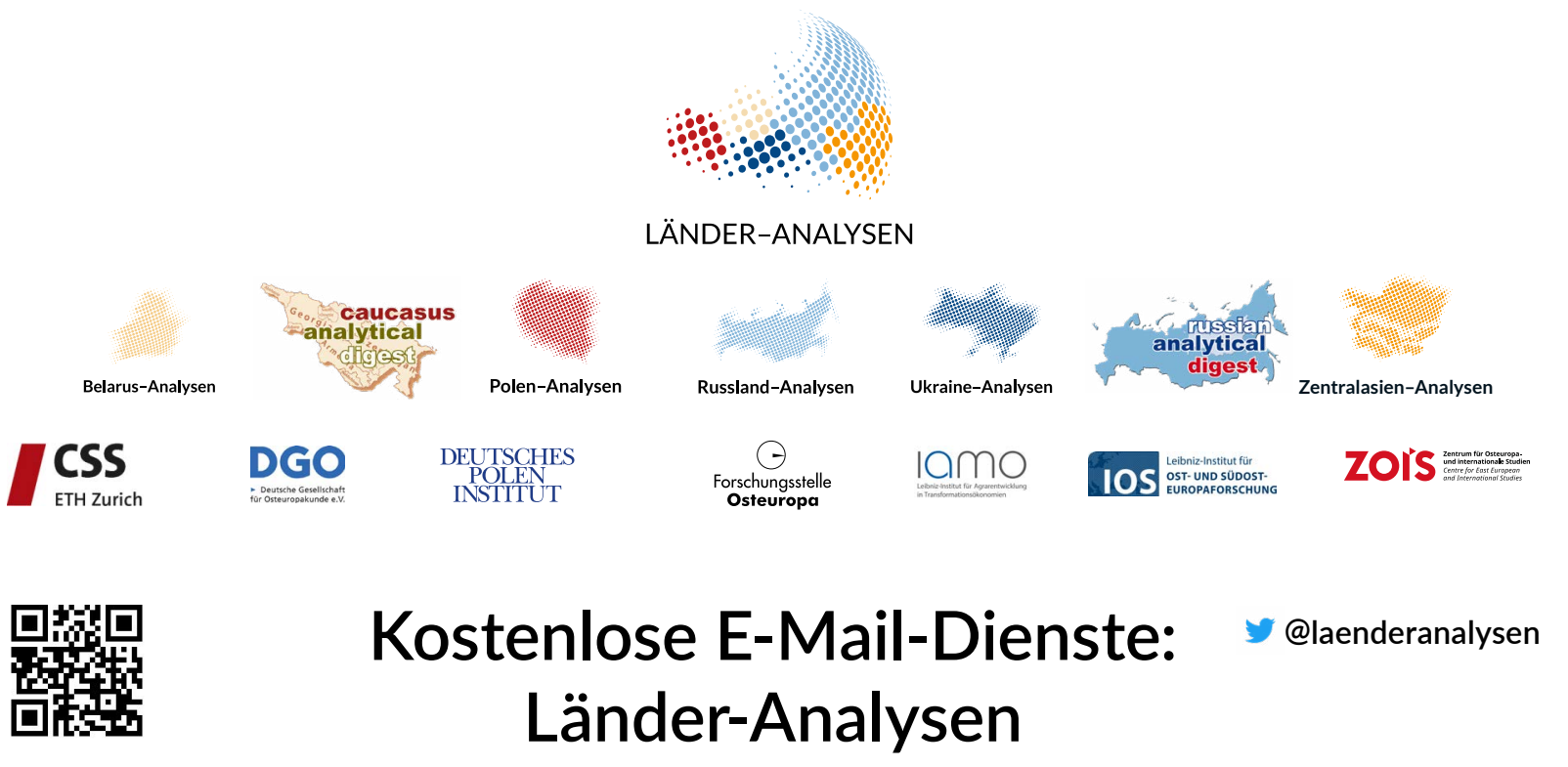

Die Länder-Analysen bieten regelmäßig im kostenlosen Abonnement kompetente Einschätzungen aktueller politischer, wirtschaftlicher, sozialer und kultureller Entwicklungen in Ostmitteleuropa und der GUS. Alle Länder-Analysen verstehen sich als Teil eines gemeinsamen Projektes, das der wissenschaftlich fundierten, allgemeinverständlich formulierten Analyse der Entwicklungen im östlichen Europa, der Offenheit für verschiedene inhaltliche Positionen und der kostenlosen und nicht-kommerziellen Information einer breit verstandenen interessierten Öffentlichkeit verpflichtet ist. Autor/innen sind internationale Fachwissenschaftler/innen und Expert/innen. Die Redaktionen der Länder-Analysen bestehen aus Wissenschaftler/innen mit langjähriger Forschungserfahrung.

Die deutschsprachigen Länder-Analysen werden gemeinsam von der Forschungsstelle Osteuropa an der Universität Bremen, dem Zentrum für Osteuropa- und internationale Studien, der Deutschen Gesellschaft für Osteuropakunde, dem Deutschen Polen-Institut, dem Leibniz-Institut für Agrarentwicklung in Transformationsökonomien und dem Leibniz-Institut für Ost- und Südosteuropaforschung herausgegeben. Die englischsprachigen Länder-Analysen erscheinen in Kooperation der Forschungsstelle Osteuropa mit dem Center for Security Studies (CSS) der ETH Zürich.

Die Länder-Analysen bieten regelmäßig Kurzanalysen zu aktuellen Themen, ergänzt um Grafiken und Tabellen sowie Dokumentationen. Zusätzlich gibt es eine Chronik aktueller Ereignisse.

\section{Belarus-Analysen}

Erscheinungsweise: zweimonatlich

Abonnement unter: http://www.laender-analysen.de/belarus/

\section{Caucasus Analytical Digest}

In englischer Sprache. Erscheinungsweise: zweimonatlich

Abonnement unter: http://www.css.ethz.ch/en/publications/cad.html

\section{Polen-Analysen}

Erscheinungsweise: zweimal monatlich

Abonnement unter: http://www.deutsches-polen-institut.de/newsletter/polen-analysen/

\section{Russland-Analysen}

Erscheinungsweise: zweimal monatlich

Abonnement unter: http://www.laender-analysen.de/russland/

\section{Russian Analytical Digest}

In englischer Sprache. Erscheinungsweise: zweimal monatlich Abonnement unter: http://www.css.ethz.ch/en/publications/rad.html

\section{Ukraine-Analysen}

Erscheinungsweise: zweimal monatlich

Abonnement unter: http://www.laender-analysen.de/ukraine/

\section{Zentralasien-Analysen}

Erscheinungsweise: zweimonatlich

Abonnement unter: http://www.laender-analysen.de/zentralasien/ 